\title{
Measures of rule interestingness in various perspectives of confirmation
}

\author{
Salvatore Greco ${ }^{1,2}$, Roman Słowiński ${ }^{3,4}$, Izabela Szczęch ${ }^{3}$ \\ ${ }^{1}$ Department of Economics \& Business, University of Catania, Italy \\ salgreco@unicit.it \\ 2 Portsmouth Business School, Centre of Operations Research and Logistics (CORL), \\ United Kingdom \\ 3 Institute of Computing Science, Poznań University of Technology, Poland \\ \{Roman.Slowinski, Izabela.Szczech\} \\ ¿cs.put.poznan.pl \\ ${ }^{4}$ Systems Research Institute, Polish Academy of Sciences, Warsaw, Poland
}

\begin{abstract}
Confirmation is a useful concept for assessing the impact of the premise on the conclusion of a rule induced from data. Interpretation of probabilistic relationships between premise and conclusion of a rule led to four mathematical formulations of confirmation, called perspectives. The logical equivalence of these perspectives and the resulting general definition of confirmation underline the known qualitative aspect of the concept of confirmation. The quantitative aspect of confirmation is handled by definitions of particular confirmation measures. In this paper, we relate the qualitative and quantitative aspects by introducing a property of monotonicity of measures with respect to left- and right-hand side probabilities defining the perspectives. This new property permits consideration of confirmation measures in association with particular perspectives. We also identify several other properties that valuable confirmation measures should possess. A particular care is devoted to discussion of behavior of confirmation measures monotonic in different perspectives with respect to symmetry properties, taking also into account two new perspectives of Bayesian confirmation. We also prove that confirmation measures monotonic in the six perspectives are exhaustive in the sense that their set is closed under transformations related to symmetry properties. Finally, we verify which confirmation measures enjoy these properties.
\end{abstract}

Keywords: Rule interestingness measures, Bayesian confirmation, Evidential support, Properties of measures 


\section{Introduction}

Confirmation is a term from philosophy of science, defined as the impact of evidence on hypothesis. For example, seeing a man with a bike helmet walk into an office can be regarded as a piece of evidence with a greater impact on a hypothesis that he came to work by bike than on a hypothesis that he came by car. The information concerning the support brought by a piece of evidence (observational data) to a hypothesis (scientific theory) is often needed in scientific research involving data analysis. Thus, naturally, philosophers of science and epistemologists have attempted to express first qualitatively, and finally quantitatively the relation between evidence and hypothesis.

The concept of confirmation has to be distinguished from the measure of confirmation. The first acknowledges qualitatively three situations: confirmation, neutrality and disconfirmation. Confirmation takes place when for a given premise $E$ and hypothesis $H$, the probability of $H$ given $E$ is greater than the probability of $H$ (i.e., $\operatorname{Pr}(H \mid E)>\operatorname{Pr}(H)$ ). Neutrality holds when $\operatorname{Pr}(H \mid E)=\operatorname{Pr}(H)$, and disconfirmation when $\operatorname{Pr}(H \mid E)<\operatorname{Pr}(H)$ [12]. On the other hand, a confirmation measure quantifies the degree of support that a given evidence $E$ brings to a hypothesis $H$. Values obtained by measures of confirmation can be positive, negative or zero, corresponding to situations of confirmation, disconfirmation or neutrality, respectively.

In this article, we mainly concentrate on measures of confirmation for potential use in the field of data mining and knowledge discovery. In particular, we regard them as means for the evaluation of rule patterns induced from datasets, where the rule's premise is seen as the evidence with an impact on the rule's conclusion, i.e., the hypothesis. The evaluation of rules is a valid and necessary step in the data mining process allowing to filter out rules that are useless or irrelevant. It is commonly done using measures of interest such as confidence, support, etc. often neglecting the profits brought by the division of values of confirmation measures into positive, negative and zero (see [2], [15], [27], [28], [34] for a survey on measures of rule interestingness). Nevertheless, users are more and more commonly interested in evaluating rules with measures that reward the rules in which the premise confirms the conclusion. It is not completely clear, however, what it means that a premise confirms the conclusion. What does the confirmation stand for and how can we quantify it?

In this article, we aim at answering the above questions by studying the concept of confirmation from different perspectives. These perspectives derive from logically equivalent formulations of the concept of Bayesian confirmation [12] and interpret in a probabilistic way relationships of confirmation type between premise and conclusion of a rule. Classically, four different perspectives, that we call Bayesian, likelihoodist, strong Bayesian, 
and strong likelihoodist, have been considered in the literature (see, e.g., [12], [28]). In this paper, we take into account two new perspectives of Bayesian confirmation, called converse Bayesian and converse likelihoodist perspectives. Besides the six logically equivalent perspectives of confirmation, we recall a general definition of confirmation in terms of a contingency table rather than in terms of probabilities [16]. We stress the fact that the general definition of confirmation has the advantage of being always defined (as opposed to the other six perspectives that become undefined in certain conditions).

While the six perspectives of confirmation underline the qualitative aspect of this concept, confirmation measures exhibit the quantitative aspect. We introduce an important link between the qualitative and quantitative aspects of confirmation, which is the property of monotonicity of measures with respect to left- and right-hand side probabilities defining the perspectives. In this way, we pass from consideration of six perspectives of confirmation to consideration of six classes of confirmation measures, each one monotonic in one of the six perspective. We prove that the confirmation measures monotonic in the six perspectives are exhaustive in the sense that their set is closed under transformations related to symmetry properties.

We also identify several other properties that valuable confirmation measures should definitely possess. The properties are analyzed and described in detail providing an insight into many necessary modifications and improvements introduced into their formulation over the time. The set of valuable properties that emerged from our analysis contains:

- property of monotonicity with respect to the number of objects satisfying the rule's premise and/or conclusion (property $\mathrm{M}$ ),

- property assuring that rules for which there are no counterexamples and no objects supporting the conclusion but not the rule's premise will obtain a higher value of a measure than rules for which such objects do exist in the dataset (weak $\mathrm{Ex}_{1}$ property),

- property of weak logicality indicating the conditions under which measures should obtain their extreme values (weak L property),

- three symmetry properties related to negation of the rule's premise and/or conclusion:

○ evidence symmetry,

○ hypothesis symmetry and

- evidence-hypothesis symmetry, being the combination of the two above symmetries.

The four perspectives of confirmation were considered already in theoretical studies [11], [28], neglecting however practical consequences of their application in data mining. In practical applications, it is desirable to assess rule interestingness using measures normalized with respect to extreme situations encountered in data. It appears, however, that some strategies to 
determining the extremes can lead to paradoxical rankings of rules. Thus, identifying which of them permits to avoid these paradoxes is a challenging task. In this paper, we undertake this task and show which of the four perspectives are the right starting points to define properties that confirmation measures should reasonably possess.

This paper builds on results presented in [22], [23], where we showed for the first time some paradoxes resulting from adoption of a particular strategy to determining the extreme situations. Moreover, this article presents a holistic view on qualitative and quantitative aspects of the concept of confirmation. The rest of the paper is organized in the following way. Section 2 presents preliminaries on data, rules and their quantitative description. Next, in Section 3, four perspectives of confirmation are thoroughly discussed. Their logical equivalence is recalled along with a general definition of confirmation. We also introduce an important property of monotonicity of a measure with respect to the left- and right-hand side probabilities defining the four perspectives. This property will be shortly called "monotonicity of a measure in a perspective". It associates confirmation measures with particular perspectives because, as proved in Section 3.2, a measure being strictly monotonic in one perspective is not strictly monotonic in other perspectives. Section 4 is devoted to properties of confirmation measures that are inspired by extreme values of confirmation. As it is shown, the strategy for determining the extreme confirmation inspired by the perspective of strong Bayesian confirmation has many practical advantages over entailment, and thus it should be used as a starting point for defining properties of measures. Consecutive subsections discuss properties already known in the literature as well as their modifications in the context of strong Bayesian confirmation. The relationship between particular properties and different perspectives of confirmation is also discussed. A particular care is devoted to discussion of behavior of confirmation measures monotonic in different perspectives with respect to symmetry properties. In this context, two new perspectives of Bayesian confirmation are introduced, showing also that together with the other four previous perspectives, they are exhaustive with respect to transformations of confirmation measure related to symmetry properties. Having established the list of desirable properties, analysis of selected measures with respect to the properties is carried out in Section 5. The paper ends with conclusions. All the proofs are deferred to an Appendix provided as on-line supplementary materials.

\section{Preliminaries about data, rules and supporting observations}

A dataset is composed of a number of observations, called objects, described by a number of variables. The objects constitute a universe $U$ from 
which rules are induced. Each rule is a consequence relation denoted by $E \rightarrow H$, read as "if $E$, then $H$ ". It consists of a premise (evidence) $E$ and a conclusion (hypothesis) $H$. In the context of a particular dataset, the relation between $E$ and $H$ may be quantified by four non-negative numbers $a, b, c$ and $d$, corresponding to all possible cases of truth and falsity of $E$ and $H$, presented in a $2 \times 2$ contingency table (see Table 1).

Table 1. Contingency table of $E$ and $H$ for rule $E \rightarrow H$

\begin{tabular}{|c|c|c|c|}
\hline & $H$ & $\neg H$ & $\sum$ \\
\hline$E$ & $a$ & $c$ & $a+c$ \\
\hline$\neg E$ & $b$ & $d$ & $b+d$ \\
\hline$\sum$ & $a+b$ & $c+d$ & $|U|$ \\
\hline
\end{tabular}

Precisely, the number of all objects in $U$ supporting both the premise and the conclusion of a rule is quantified by $a ; b$ reflects the number of objects for which the premise in not satisfied, but the conclusion is, etc.

Using $a, b, c$ and $d$ is common and intuitive for data mining techniques since all observations are collected in an information table describing each object by a set of variables. However, $a, b, c$ and $d$ can also be used to estimate probabilities, e.g., $\operatorname{Pr}(E)=(a+c) /|U| \quad$ or $\quad \operatorname{Pr}(H)=(a+b) /|U|$, $\operatorname{Pr}(H \mid E)=a /(a+c)$ (which, however, is only defined when $a+c>0)$.

The notation based on a, b, c and d can be effectively used for defining such interestingness measures as confidence $\operatorname{conf}(H, E)=a /(a+c)$, support $\sup (H, E)=a$, etc. Observe, that the domain of both confidence and support is non-negative, therefore they are unable to express disconfirmation and cannot be treated as measures of confirmation. The scale of confidence or support is not helpful for filtering out rules in which the rule's premise has a negative impact on the rule's conclusion. The profits from using confirmation measures, on the other hand, rest upon the scale showing immediately (by negative values) disconfirmatory rules. Among popular measures of confirmation there is measure $D(H, E)=\operatorname{Pr}(H \mid E)-\operatorname{Pr}(H)=a /(a+c)-(a+b) /|U|$ defended among others in [7], measure $N(H, E)=\operatorname{Pr}(E \mid H)-\operatorname{Pr}(E \mid \neg H)=a /(a+b)-c /(c+d)$ supported by [33], or measure $F(H, E)=[\operatorname{Pr}(E \mid H)-\operatorname{Pr}(E \mid \neg H)] /[\operatorname{Pr}(E \mid H)+\operatorname{Pr}(E \mid \neg H)]$

$=(a d-b c) /(a d+b c+2 a c)$ advocated for in [25]. Other interesting confirmation measures shall be presented in Section 5.

\section{Four perspectives of confirmation}

A common expectation with respect to the behavior of interestingness measures used for evaluation of rules is that they obtain: 
- values $>0$ when the premise of a rule confirms its conclusion,

- values $=0$ when the rule's premise and conclusion are neutral to each other,

- values $<0$ when the premise disconfirms the conclusion.

Such requirements are referred to (informally) as the property of confirmation and thus, measures acting according to them are called confirmation measures.

A commonly used definition of confirmation property, called Bayesian confirmation, requires that an interestingness measure $c(H, E)$ satisfies the following conditions:

$$
c(H, E)\left\{\begin{array}{l}
>0 \text { if } \operatorname{Pr}(H \mid E)>\operatorname{Pr}(H), \\
=0 \text { if } \operatorname{Pr}(H \mid E)=\operatorname{Pr}(H), \\
<0 \text { if } \operatorname{Pr}(H \mid E)<\operatorname{Pr}(H) .
\end{array}\right.
$$

The property of Bayesian confirmation identifies confirmation with an increase in the probability of the conclusion provided by the premise, neutrality with the lack of influence of the premise on the probability of conclusion, and finally disconfirmation with a decrease of probability of the conclusion imposed by the premise [4]. In the literature [12], [28], such understanding of confirmation is sometimes also called incremental Bayesian confirmation, as opposed to the absolute confirmation which assumes that $E$ confirms $H$, if some kind of a threshold $\mathrm{k} \in(0,1)$ is surpassed by the conditional probability of $H$ given $E$. This article however shall not cover the absolute confirmation.

It is important to stress that the Bayesian confirmation is not the only definition of property of confirmation. In the literature (see [12], [28]), there are three other ways of expressing that $E$ confirms $H$ :

- $\operatorname{Pr}(H \mid E)>\operatorname{Pr}(H \mid \neg E)$,

- $\operatorname{Pr}(E \mid H)>\operatorname{Pr}(E)$,

- $\operatorname{Pr}(E \mid H)>\operatorname{Pr}(E \mid \neg H)$.

This gives four perspectives in which confirmation can be considered. Below, we propose a way of systematizing them, pointing out the differences between them and recalling their logical equivalence.

To better distinguish the perspectives of confirmation let us call them in the following way (for clarity of the presentation, in brackets we put only the conditions under which a measure should obtain positive values, as the conditions for neutrality and negative values are analogously formed):

(i) Bayesian confirmation $(\operatorname{Pr}(H \mid E)>\operatorname{Pr}(H))$,

(ii) strong Bayesian confirmation $(\operatorname{Pr}(H \mid E)>\operatorname{Pr}(H \mid \neg E))$,

(iii) likelihoodist confirmation $(\operatorname{Pr}(E \mid H)>\operatorname{Pr}(E))$,

(iv) strong likelihoodist confirmation $(\operatorname{Pr}(E \mid H)>\operatorname{Pr}(E \mid \neg H))$. 
Those perspectives can be naturally grouped into couples reaching to the debate between Bayesians and likelihoodists about confirmation's probabilistic interpretation [14]. Let us note that rule $E \rightarrow H$ in the Bayesian viewpoint, corresponds to rule $H \rightarrow E$ in the likelihoodist approach.

All those four perspectives have different philosophical background and motivations. They emphasize different faces of confirmation:

- the Bayesian confirmation states that $E$ confirms $H$ if $H$ is more probable with $E$ rather than without $E$, where "without $E$ " means without knowing if $E$ or $\neg E$ is true,

- the strong Bayesian confirmation stresses that $E$ confirms $H$ if $H$ is more probable with $E$ rather than with $\neg E$,

- the likelihoodist confirmation says that $E$ confirms $H$ if $E$ is more probable with $H$ rather than without $H$, where "without $H$ " means without knowing if $H$ or $\neg H$ is true,

- finally, the strong likelihoodist confirmation states that $E$ confirms $H$ if $E$ is more probable with $H$ rather than with $\neg H$.

To provide an interpretation of the four perspectives of confirmation let us use an illustrative example, in which the premise $E$ is the evidence that a patient suffered from a fever and the conclusion $H$ reflects that the patient had a flu. Then:

- in case of Bayesian confirmation (i) if flu is more probable with fever rather than without knowing whether the fever occurred or not, then fever confirms flu,

- in case of strong Bayesian confirmation (ii) if flu is more probable with fever rather than with no fever, than fever confirms flu,

- in case of likelihoodist confirmation (iii) if fever is more probable with flu rather than without knowing whether the flu occurred of not, then fever confirms flu,

- in case of strong likelihoodist confirmation (iv) if fever is more probable with flu rather than with no flu, then fever confirms flu.

Let us stress that the difference between those four perspectives of confirmation does not only come from different philosophical backgrounds, motivations or interpretations. The particular formulations in terms of probabilities or frequencies involving $a, b, c$ and $d$ also result in differences with respect to undefined situations they may lead to.

In particular, the perspective of Bayesian confirmation in terms of probabilities is formulated as $\operatorname{Pr}(H \mid E)>\operatorname{Pr}(H)$, which can be estimated by the non-negative numbers as $a /(a+c)>(a+b) /|U|$. Clearly, such formulation requires that $\operatorname{Pr}(E) \neq 0$ (or more precisely $\operatorname{Pr}(E)>0$ ) or equivalently $a+c \neq 0$ (or more precisely $a+c>0$ ).

The perspective of strong Bayesian confirmation has even stronger requirements, since the formulation $\operatorname{Pr}(H \mid E)>\operatorname{Pr}(H \mid \neg E$ ) (or equivalently 
$a /(a+c)>b /(b+d))$ in order to be defined wants that $\operatorname{Pr}(E) \neq 0$ and $\operatorname{Pr}(\neg E) \neq 0$ (or equivalently $a+c \neq 0$ and $b+d \neq 0$ ).

Analogous considerations for the perspectives of likelihoodist confirmation and strong likelihoodist confirmation lead to requiring that $\operatorname{Pr}(H) \neq 0$ (or equivalently $a+b \neq 0$ ) or that $\operatorname{Pr}(H) \neq 0$ and $\operatorname{Pr}(\neg H) \neq 0$ (or equivalently $a+b \neq 0$ and $c+d \neq 0$ ), respectively.

Since the different perspectives are undefined in different situations (in different points of the four dimensional domain, i.e., domain based on $a, b, c$, $d$ ), they are not equivalent in general; they are different. For example, the perspective of strong Bayesian confirmation would result in an undefined value (analogous to an "I don't know" answer) when $b+d=0$, while at the same time the perspective of Bayesian confirmation could be defined, pointing out the situation of neutrality.

\subsection{Logical equivalence of four perspectives of confirmation}

As the above introduction shows, the four perspectives of confirmation should be regarded as different, alternative ways of formalizing this concept. Nevertheless, it is important to notice that the four perspectives are logically equivalent, provided they do not lead to undefined values. By logical equivalence we understand that the conditions which need to be satisfied to switch between positive, zero and negative values are the same for all the perspectives. Thus, they are not the same, but they "switch" in the same situations, which we will demonstrate below.

Let us observe that the situation of confirmation with respect to Bayesian confirmation is represented by the following inequality: $\operatorname{Pr}(H \mid E)>\operatorname{Pr}(H)$. Using the non-negative numbers $a, b, c$ and $d$, it can be expressed as $a /(a+c)>(a+b) /|U|$ (of course, we require that $a+c \neq 0$ ). Simple mathematical transformations show that $a /(a+c)>(a+b) /|U|$ iff $a|U|>(a+b)(a+c)$, which can be further simplified to $a d-b c>0$. Thus, provided that Bayesian confirmation is defined (i.e., $a+c \neq 0$ ), the $a d-b c\{>,=,<\} 0$ are the conditions for switching between situation of confirmation, neutrality and disconfirmation, respectively.

Regarding the strong Bayesian confirmation, the situation of confirmation is represented as $\operatorname{Pr}(H \mid E)>\operatorname{Pr}(H \mid \neg E)$, which can be also expressed as $a /(a+c)>b /(b+d)$ (of course, we require that $a+c \neq 0$ and $b+d \neq 0)$. Simple mathematical transformations show that $a /(a+c)>b /(b+d)$ iff $a(b+d)>b(a+c)$, which can be further simplified to $a d-b c>0$. Thus, provided that the definition of the strong Bayesian confirmation is defined (i.e., $a+c \neq 0$ and $b+d \neq 0$ ), $a d-b c\{>,=,<\} 0$ are the conditions for switching 
between situation of confirmation, neutrality and disconfirmation, respectively.

Analogous transformations can be performed for likelihoodist and strong likelihoodist confirmations, showing that again the $a d-b c\{>,=,<\} 0$ are the conditions for switching between situation of confirmation, neutrality and disconfirmation, respectively.

Summing up, one can formulate a general conclusion, that there are four (i) - (iv) alternative, different perspectives of confirmation, but, provided they are defined, they all boil down to the following general definition of confirmation expressed in terms of the non-negative $a, b, c$ and $d$, as in [22] (page 5) and [16] (Proposition 1):

$$
c(H, E)\left\{\begin{array}{l}
>0 \text { if } a d-b c>0, \\
=0 \text { if } a d-b c=0, \\
<0 \text { if } a d-b c<0 .
\end{array}\right.
$$

Coming back to the interpretation of $a, b, c, d$ in terms of probabilities, (2) is equivalent to:

$$
c(H, E)\left\{\begin{array}{l}
>0 \text { if } \operatorname{Pr}(H \wedge E) \operatorname{Pr}(\neg H \wedge \neg E)>\operatorname{Pr}(H \wedge \neg E) \operatorname{Pr}(\neg H \wedge E), \\
=0 \text { if } \operatorname{Pr}(H \wedge E) \operatorname{Pr}(\neg H \wedge \neg E)=\operatorname{Pr}(H \wedge \neg E) \operatorname{Pr}(\neg H \wedge E), \\
<0 \text { if } \operatorname{Pr}(H \wedge E) \operatorname{Pr}(\neg H \wedge \neg E)<\operatorname{Pr}(H \wedge \neg E) \operatorname{Pr}(\neg H \wedge E) .
\end{array}\right.
$$

The logical equivalence of Bayesian confirmation, strong Bayesian confirmation, likelihoodist confirmation and strong likelihoodist confirmation with the " $a d-b c$ " formulation is true provided that none of the (i)-(iv) formulations is undefined, which means that all the following sums: $a+c, b+d$, $a+b$ and $c+d$ are non-zero.

The above general definition of confirmation has the advantage over the (i)-(iv) perspectives of never being undefined. The fact that there is no denominator in it, guarantees that for any dataset, and thus any particular contingency table with $a, b, c$ and $d$, definition (2) determines whether we are in the situation of confirmation, neutrality of disconfirmation. On the other hand, working with Bayesian confirmation, strong Bayesian confirmation, likelihoodist confirmation or strong likelihoodist confirmation we can also obtain the undesirable undefined situations (when $a+c=0$, or $b+d=0$, or $a+b=0$, or $c+d=0$ ).

What makes the matter even worse, is that the four perspectives are undefined in different situations, i.e., one perspective can be undefined while the other can be defined. This could be definitely confusing for practitioners who expect a clear answer: confirmation, neutrality or disconfirmation in the 
whole domain. The general definition of confirmation is always defined and provides the neutral answer whenever undefined situations occur for the other perspectives, i.e., when $a+c=0$, or $b+d=0$, or $a+b=0$, or $c+d=0$. For example, in case when $a+c=0$, we can conclude that $a=c=0$ (since all values $a, b, c, d$ are assumed to be non-negative), which boils the formula $a d-b c$ to zero expressing the neutrality. The general definition of confirmation is thus a kind of brace for the other formulations that can be especially useful when working with real life data sets.

\subsection{Desirable property of monotonicity of confirmation measures with respect to left- and right-hand side probabilities of the four perspectives of confirmation}

The four logically equivalent perspectives of confirmation suggest four kinds of monotonicity for confirmation measures. In fact,

- Bayesian confirmation $(\operatorname{Pr}(H \mid E)>\operatorname{Pr}(H))$ suggests that a confirmation measure $c(H \mid E)$ should be non-decreasing with respect to $\operatorname{Pr}(H \mid E)$ and non-increasing with $\operatorname{Pr}(H)$,

- $\quad$ strong Bayesian confirmation $(\operatorname{Pr}(H \mid E)>\operatorname{Pr}(H \mid \neg E))$ suggests that a confirmation measure $c(H \mid E)$ should be non-decreasing with respect to $\operatorname{Pr}(H \mid E)$ and non-increasing with $\operatorname{Pr}(H \mid \neg E)$,

- likelihoodist confirmation $(\operatorname{Pr}(E \mid H)>\operatorname{Pr}(E))$ suggests that a confirmation measure $c(H \mid E)$ should be non-decreasing with respect to $\operatorname{Pr}(E \mid H)$ and non-increasing with $\operatorname{Pr}(E)$,

- strong likelihoodist confirmation $(\operatorname{Pr}(E \mid H)>\operatorname{Pr}(E \mid \neg H))$ suggests that a confirmation measure $c(H \mid E)$ should be non-decreasing with respect to $\operatorname{Pr}(E \mid H)$ and non-increasing with $\operatorname{Pr}(E \mid \neg H)$.

Indeed, in all these perspectives, one can switch from a situation of disconfirmation to a situation of confirmation if the left-hand side probability increases (while the right-hand side probability does not increase) or the righthand side probability decreases (while the left-hand side probability does not decrease). It is thus reasonable to require that the increase of the left-hand side probability or decrease of the right-hand side probability should not decrease the confirmation $c(H, E)$ that quantifies the support that evidence $E$ gives to hypothesis $H$.

Coming back to the example where the premise $E$ is the evidence that a patient has a fever, and the conclusion $H$ is the hypothesis that the patient has a flu, we can say that for a confirmation measure

- being monotonic with respect to left- and right-hand side probabilities of the Bayesian perspective, the greater the probability of flu in case of 
fever, and the smaller the probability of flu, the more the fever confirms the flu,

- being monotonic with respect to left- and right-hand side probabilities of the strong Bayesian perspective, the greater the probability of flu in case of fever, and the smaller the probability of flu in case of no fever, the more the fever confirms the flu,

- being monotonic with respect to left- and right-hand side probabilities of the likelihoodist perspective, the greater the probability of fever in case of flu, and the smaller the probability of fever, the more the fever confirms the flu,

- being monotonic with respect to left- and right-hand side probabilities of the strong likelihoodist perspective, the greater the probability of fever in case of flu, and the smaller the probability of fever in case of no flu, the more the fever confirms the flu.

A formal definition of the monotonicity of a measure with respect to leftand right-hand side probabilities of particular perspective, is as follows.

Definition 1. A confirmation measure $c(H, E)$ is monotonic with respect to left- and right-hand side probabilities of a particular perspective if, taking into account left- and right-hand side probabilities of the perspective, there exists $f:[0,1] \times[0,1] \rightarrow \Re$ non-decreasing with the first argument and non-increasing with the second argument, with $f(x, x)=0$ for all $x \in[0,1]$, such that $c(H, E)=f\left(\operatorname{Pr}_{\mathrm{L}}, \operatorname{Pr}_{\mathrm{R}}\right)$ where $\operatorname{Pr}_{\mathrm{L}}$ is the left-hand side probability and $\operatorname{Pr}_{\mathrm{R}}$ is the right-hand side probability of the considered perspective.

For the sake of simplicity, instead of writing that a particular confirmation measure is monotonic with respect to left- and right-hand side probabilities of a given perspective we will shortly write that a particular confirmation measure is monotonic in a given perspective.

Examples of typical confirmation measures monotonic in particular perspectives include:

- a measure monotonic in the Bayesian perspective: $D(H, E)=\operatorname{Pr}(H \mid E)-\operatorname{Pr}(H)=a /(a+c)-(a+b) /|U|$,

- a measure monotonic in the strong Bayesian perspective: $S(H, E)=\operatorname{Pr}(H \mid E)-\operatorname{Pr}(H \mid \neg E)=a /(a+c)-b /(a+b)$,

- a measure monotonic in the likelihoodist perspective: $M(H, E)=\operatorname{Pr}(E \mid H)-\operatorname{Pr}(E)=a /(a+b)-(a+c) /|U|$,

- a measure monotonic in the strong likelihoodist perspective: $N(H, E)=\operatorname{Pr}(E \mid H)-\operatorname{Pr}(E \mid \neg H)=a /(a+b)-c /(c+d)$.

Consider now a rule $E \rightarrow H$ in two scenarios for which the values of $a, b, c$ and $d$ can change (e.g., some objects regarded as counterexamples in 
scenario 1 are regarded as positive examples in scenario 2). The two scenarios correspond to contingency tables in Table 2 and Table 3 , respectively.

Table 2. Contingency table for scenario 1

\begin{tabular}{|c|c|c|c|}
\hline & $H_{1}$ & $\neg H_{1}$ & $\sum$ \\
\hline$E_{1}$ & $a_{1}=10$ & $c_{1}=2$ & $a_{1}+c_{1}=12$ \\
\hline$\neg E_{1}$ & $b_{1}=10$ & $d_{1}=78$ & $b_{1}+d_{1}=88$ \\
\hline$\sum$ & $a_{1}+b_{1}=20$ & $c_{1}+d_{1}=80$ & $|U|=100$ \\
\hline
\end{tabular}

Table 3. Contingency table for scenario 2

\begin{tabular}{|c|c|c|c|}
\hline & $H_{2}$ & $\neg H_{2}$ & $\sum$ \\
\hline$E_{2}$ & $a_{2}=6$ & $c_{2}=1$ & $a_{2}+c_{2}=7$ \\
\hline$\neg E_{2}$ & $b_{2}=13$ & $d_{2}=80$ & $b_{2}+d_{2}=93$ \\
\hline$\sum$ & $a_{2}+b_{2}=19$ & $c_{2}+d_{2}=81$ & $|U|=100$ \\
\hline
\end{tabular}

Observe that

$$
\begin{aligned}
& \operatorname{Pr}\left(H_{1} \mid E_{1}\right)=\frac{a_{1}}{a_{1}+c_{1}}=0.833, \quad \operatorname{Pr}\left(H_{1}\right)=\frac{a_{1}+b_{1}}{a_{1}+b_{1}+c_{1}+d_{1}}=0.2, \\
& \operatorname{Pr}\left(H_{2} \mid E_{2}\right)=\frac{a_{2}}{a_{2}+c_{2}}=0.857, \quad \operatorname{Pr}\left(H_{2}\right)=\frac{a_{2}+b_{2}}{a_{2}+b_{2}+c_{2}+d_{2}}=0.19,
\end{aligned}
$$

so that

$$
\frac{a_{2}}{a_{2}+c_{2}}>\frac{a_{1}}{a_{1}+c_{1}} \text { and } \frac{a_{2}+b_{2}}{a_{2}+b_{2}+c_{2}+d_{2}}<\frac{a_{1}+b_{1}}{a_{1}+b_{1}+c_{1}+d_{1}} \text {. }
$$

Thus, by definition, for any confirmation measure monotonic in the Bayesian perspective we get $c\left(H_{2}, E_{2}\right) \geq c\left(H_{1}, E_{1}\right)$, since $\operatorname{Pr}(H \mid E)$ increased and $\operatorname{Pr}(H)$ decreased, while passing from scenario 1 to scenario 2 .

Indeed, we get $D\left(H_{1}, E_{1}\right)=0.633$ and $D\left(H_{2}, E_{2}\right)=0.667$ so clearly $D\left(H_{2}, E_{2}\right)>D\left(H_{1}, E_{1}\right)$. However considering the other three confirmation measures given above we get:

- $S\left(H_{1}, E_{1}\right)=0.720$ and $S\left(H_{2}, E_{2}\right)=0.717$ so that $S\left(H_{2}, E_{2}\right)<S\left(H_{1}, E_{1}\right)$;

- $M\left(H_{1}, E_{1}\right)=0.38$ and $M\left(H_{2}, E_{2}\right)=0.246$ so that $M\left(H_{2}, E_{2}\right)<M\left(H_{1}, E_{1}\right)$;

- $\quad N\left(H_{1}, E_{1}\right)=0.475$ and $N\left(H_{2}, E_{2}\right)=0.303$ so that $N\left(H_{2}, E_{2}\right)<N\left(H_{1}, E_{1}\right)$.

This shows that confirmation measures $S(H, E), M(H, E)$ and $N(H, E)$ are not monotonic in the Bayesian perspective. 
Similar examples can be built to prove that $D(H, E), M(H, E)$ and $N(H, E)$ are not monotonic in the strong Bayesian perspective, $D(H, E), S(H, E)$ and $N(H, E)$ are not monotonic in the likelihoodist perspective and $D(H, E), S(H, E)$ and $M(H, E)$ are not monotonic in the strong likelihoodist perspective.

In the following theorem, we will show that the observation obtained above for particular measures is more general, provided we assume strict monotonicity with respect to left- and right-hand side probabilities defining a perspective of confirmation (i.e., strict monotonicity of a measure in a perspective of confirmation). See the Appendix for the proofs of all Theorems and Lemmas. The Appendix is provided as the on-line supplementary materials.

Theorem 1. A confirmation measure being strictly monotonic in a given perspective, $c(H, E)=f\left(\operatorname{Pr}_{\mathrm{L}}, \operatorname{Pr}_{\mathrm{R}}\right)$ with $f$ being strictly increasing in the first argument and strictly decreasing in the second argument, is not strictly monotonic in the other perspectives.

\subsection{Desirable property of monotonicity of confirmation measures with respect to left- and right-hand side probabilities of the general definition of confirmation}

Finally, let us also consider the monotonicity of a confirmation measure with respect to the left- and right-hand side probabilities for the general definition of confirmation. In case of the general definition of confirmation (3), there are four probabilities - two on the left-hand side and two on the right-hand side. Then, one can switch from a situation of disconfirmation to a situation of confirmation if the product on the left-hand side increases or the product on the right-hand side decreases. It is thus reasonable again to require that the increase of the left-hand side product or decrease of the right-hand side product should not decrease the confirmation $c(H, E)$ that quantifies the support that evidence $E$ gives to hypothesis $H$. Confirmation measures satisfying such requirement shall be regarded as monotonic in the general definition of confirmation. Later, in Section 4.1, we show that such monotonicity of measures in the general definition of confirmation boils down to the property of monotonicity $\mathrm{M}$.

On the basis of above discussion on monotonicity of confirmation measures in different perspectives of confirmation (Section 3.2), it is very appealing to define a confirmation measure $f(a, b, c, d)$ monotonic in the general definition of confirmation. The simplest measures of this type would have the form:

$$
f_{\text {basic }}(a, b, c, d)=a d-b c \text {. }
$$


Observe also that a very reasonable property one could expect from a function $f(a, b, c, d)$ is its homogeneity of degree 0 which means that if all arguments of $f$ are multiplied by a positive constant $\lambda$, then its value should not change, i.e.,

$f(\lambda a, \lambda b, \lambda c, \lambda d)=f(a, b, c, d)$.

This means that the confirmation should depend on the proportion of the elements in the classes of the different combinations of truth and falsity of $E$ and $H$ and not on their absolute values. Indeed, taking $\lambda=1 /|U|$, we have

$$
\begin{aligned}
& f(a, b, c, d)=f_{G}\left(\frac{a}{|U|}, \frac{b}{|U|}, \frac{c}{|U|}, \frac{d}{|U|}\right)= \\
& =f[\operatorname{Pr}(E \wedge H), \operatorname{Pr}(\neg E \wedge H), \operatorname{Pr}(E \wedge \neg H), \operatorname{Pr}(\neg E \wedge \neg H)]
\end{aligned}
$$

Observe that the confirmation measure $f_{\text {basic }}(a, b, c, d)=a d-b c$ does not satisfy the property of homogeneity of degree 0 . To get this result one could "normalize" the quantity. Many association indices follow this logic, such as the Pearson Phi index [36], [44]:

$$
f_{\text {Pearson }}(a, b, c, d)=\frac{a d-b c}{\sqrt{(a+b)(a+c)(b+c)(c+d)}}
$$

or the Yule Q index [43]:

$$
f_{\text {Yule }}(a, b, c, d)=\frac{a d-b c}{a d+b c} .
$$

Both the two above indices, however, have the disadvantage of not being defined for some values of their arguments because the denominator is null.

To avoid this, one could consider some normalization of the quantity $a d-b c$ for which the denominator is always different from zero. This is the case of the Michael coefficient of association [30]:

$$
f_{\text {Michael }}(a, b, c, d)=\frac{4(a d-b c)}{(a+d)^{2}+(b+c)^{2}} .
$$

Unfortunately, computing partial derivatives with respect to its arguments one can see that this measure does not respect monotonicity $M$ introduced in [18] and discussed in the next Section 4.1, i.e., it is not non-decreasing with respect to $a$ and $d$ and non-increasing with respect to $b$ and $c$ in the whole domain and thus, strictly speaking, it cannot be considered a measure monotonic in the general definition of confirmation. On the contrary, the Pearson Phi index and the Yule Q index respect monotonicity of $a, b, c$ and $d$ in the whole domain. Thus, an interesting problem remains open: is there a confirmation measure obtained by normalizing the quantity $a d-b c$ that is 
defined in the whole domain (in the sense that the denominator is never null) and that respects the monotonicity of $a, b, c$ and $d$ in the whole domain?

\section{Properties of confirmation measures inspired by extreme values of confirmation}

To handle the plurality of confirmation measures, various properties have been defined and studied (e.g., [4], [7], [15], [18], [40], [42]). Measures possessing them are regarded as responding to the user's expectations and thus are more useful in practical applications. Many properties proposed in the literature concern cases in which measures should obtain their extreme values (e.g., [6], [7], [12]) in result of acquiring knowledge about $E$ for the conclusion $H$. Intuitively, an extreme value of confirmation occurs in case of logical entailment of the conclusion $H$ by the premise $E$, i.e., when $E \mid=H$. This means that when $\operatorname{Pr}(H \mid E)=1$, i.e., when there are no counterexamples to the evaluated rule $(c=0)$, the value of a confirmation measure is expected to be extreme. Such a strategy is applied for properties $\mathrm{Ex}_{1}$, logicality, or in discussions about symmetry properties, when it is argued that a rule "if a drawn card is a Jack, then it is a face card" is a conclusively confirmatory rule, and thus " $100 \%$ confirmatory" [6], [7], [12].

However, an important question that we need to ask is whether such a dependence of maximal confirmation only on $\operatorname{Pr}(H \mid E)$ is sufficient for defining desirable properties of measures. In fact, during the rule evaluation we are interested in finding out how much brings $E$ to our knowledge about $H$. Thus, we need some kind of reference to $H$ itself. Let us consider the two scenarios described in Table 4.

Table 4. Contingency tables for scenario 3 and 4

\begin{tabular}{|c|c|c|c|}
\hline & $H_{3}$ & $\neg H_{3}$ & $\sum$ \\
\hline$E_{3}$ & $a_{3}=100$ & $c_{3}=0$ & $a_{3}+c_{3}=100$ \\
\hline$\neg E_{3}$ & $b_{3}=99$ & $d_{3}=1$ & $b_{3}+d_{3}=100$ \\
\hline$\sum$ & $a_{3}+b_{3}=199$ & $c_{3}+d_{3}=1$ & $|U|=200$ \\
\hline & & & \\
\hline & $H_{4}$ & $\neg H_{4}$ & $\sum$ \\
\hline$E_{4}$ & $a_{4}=99$ & $c_{4}=1$ & $a_{4}+c_{4}=100$ \\
\hline$\neg E_{4}$ & $b_{4}=0$ & $d_{4}=100$ & $b_{4}+d_{4}=100$ \\
\hline$\sum$ & $a_{4}+b_{4}=99$ & $c_{4}+d_{4}=101$ & $|U|=200$ \\
\hline
\end{tabular}


In the case of scenario 3, the value of confirmation is thought to be extreme as there are no counterexamples: $c_{3}=0$ and $\operatorname{Pr}\left(H_{3} \mid E_{3}\right)=1$ (we have entailment). The number of objects supporting $H_{3}$ is very large in scenario 3; the conclusion $\mathrm{H}_{3}$ could be regarded as a majority class in a highly imbalanced dataset. In scenario $3, \operatorname{Pr}\left(H_{3}\right)=199 / 200$, we can thus conclude that knowing that the premise $E_{3}$ occurred does not bring much to our knowledge about $H_{3}$. On the other hand, in case of scenario 4 we have one counterexample $\left(c_{4}=1\right)$ and we cannot conclude that we have entailment $\left(\operatorname{Pr}\left(H_{4} \mid E_{4}\right)<1\right)$. However, the premise has brought more to our knowledge about the conclusion in scenario 4 rather than in scenario 3. Thus, the strategy based on using the entailment in order to distinguish situations when confirmation should be extreme is not the best choice.

In the light of the above observation, let us now exploit a strategy inspired by the formulation of strong Bayesian confirmation instead (analogical considerations can be carried out for the strong likelihoodist perspective). Strong Bayesian confirmation has already proved to bring satisfactory results in such areas of research as computer science [3], [18] or applications of computer science in pharmacy and chemistry [35]. It is an alternative, logically equivalent formulation of the property of confirmation, based on relation between two probabilities: $\operatorname{Pr}(H \mid E)=a /(a+c)$ and $\operatorname{Pr}(H \mid \neg E)=b /(b+d)$. Let us remind, that for a given rule $E \rightarrow H$, an interestingness measure defined according to the strong Bayesian confirmation perspective should express the credibility of the following proposition: $H$ is satisfied more frequently when $E$ is satisfied, rather than when $\neg E$ is satisfied. Such formulation allows us to determine the most beneficial passing from $\operatorname{Pr}(H \mid \neg E)$ to $\operatorname{Pr}(H \mid E)$ due to $E$, which, according to our strategy, should be regarded as the extreme strong Bayesian confirmation. When $\operatorname{Pr}(H \mid \neg E)=0$ (i.e., when $b=0$ ) and $\operatorname{Pr}(H \mid E)=1$ (i.e., when $c=0$ ) we gain most profits on knowledge about $H$ from occurrence of $E$, instead of $\neg E$. Thus, we postulate to associate the maximum of confirmation with the situations when $b=c=0$ and thus exploit the strategy inspired by the strong Bayesian confirmation instead of the strategy inspired by the entailment, when defining desirable properties of confirmation measures. Let us note, moreover, that using strong Bayesian confirmation we are also in track with the general definition of confirmation, as it assumes that the confirmation has the chance of being extreme when $b=c=0$.

In the spirit of this section, let us observe that also for confirmation measures monotonic in the general definition of confirmation, maximal confirmation should be reached when $b=c=0$. However, differently from the strong Bayesian confirmation, for the value of such measures the proportion between $a$ and $d$ also plays a role. Therefore it would be reasonable to assign the maximum value of confirmation when the product $a d$ is maximal under fixed cardinality of the universe $U$. This is obtained in case $a=d=|U| / 2$, in 
which case $a d=|U|^{2} / 4$. Of course, symmetrically, the minimum value should be assigned when the product $b c$ is maximal under fixed cardinality of the universe $U$, which is the case when $b=c=|U| / 2$, in which case $b c=|U|^{2} / 4$. This is the case of the above mentioned Michael coefficient of association, as well as of a confirmation measure obtained putting this maximal value at the denominator of the value $a d-b c$, that is

$$
f_{\text {normalized }}(a, b, c, d)=\frac{4(a d-b c)}{(a+b+c+d)^{2}} .
$$

Let us observe that this formulation corresponds to confirmation measure $C(H, E)[4]$ (see also Section 5) as $4 C(H, E)=f_{\text {normalized }}(a, b, c, d)$. Unfortunately, calculating the partial derivatives with respect to $a, b, c$ and $d$ one can see that also this measure is not non-decreasing with respect to $a$ and $d$ and nonincreasing with respect to $b$ and $c$. Thus, it does not permit to give a positive answer to the question whether there exists a confirmation measure obtained normalizing the quantity $a d-b c$ that is defined in the whole domain (in the sense that the denominator is never null) and that is monotonic in the general definition of confirmation, as well as respects the monotonicity $\mathrm{M}$ (see Section 4.1) with respect to $a, b, c$ and $d$ in the whole domain.

Previous considerations about properties of confirmation measures by authors like Eells and Fitelson [7], Fitelson [12], [13], Crupi, Tentori and Gonzalez [6] have been based on the understanding of Bayesian confirmation as a generalization of logical entailment, and on the assumption that entailment of $H$ by $E$ should be followed by extreme confirmation. Their propositions of properties, including symmetry properties, $\mathrm{Ex}_{1}$ and logicality L, represent a strategy inspired by the entailment. Below, we discuss a number of desirable properties based on the proposed strategy inspired by the strong Bayesian confirmation perspective. Some of them are modifications of properties based on those related to entailment.

\subsection{Property of monotonicity $M$}

Greco, Pawlak and Słowiński have considered in [18] confirmation measures with respect to their usefulness for evaluation of decision rules. According to [18], a measure should enjoy a property, called property of monotonicity $\mathrm{M}$. Formally, a confirmation measure $c(H, E)=f(a, b, c, d)$ has the property $\mathrm{M}$ if and only if it is a function:

- non-decreasing with respect to $a$, and

- non-increasing with respect to $b$, and

- non-increasing with respect to $c$, and

- non-decreasing with respect to $d$. 
The property $\mathrm{M}$ with respect to $a$ (or, analogously, with respect to $d$ ) means that any evidence in which the premise $E$ and the conclusion $H$ (or, analogously, $\neg E$ and $\neg H$ ) hold together does not decrease the confirmation of the rule $E \rightarrow H$. On the other hand, the property $\mathrm{M}$ with respect to $b$ (or, analogously, with respect to $c$ ) means that any evidence in which $\neg E$ and $H$ holds (or, analogously, $E$ and $\neg H$ hold) does not increase the confirmation of the rule $E \rightarrow H$.

Let us observe that such formulation of the property of monotonicity $\mathrm{M}$ reflects the monotonicity in the strong Bayesian perspective, the strong likelihoodist perspective as well as the general definition of confirmation (2). Since the strong Bayesian confirmation is based on $\operatorname{Pr}(H \mid E)=a /(a+c)$ and $\operatorname{Pr}(H \mid \neg E)=b /(b+d)$, naturally, the increase in $a$ and $d$ accompanied by a decrease in $b$ and $c$ widens the difference between $\operatorname{Pr}(H \mid E)$ and $\operatorname{Pr}(H \mid \neg E)$, making the first bigger and the latter smaller in value. With respect to the strong likelihoodist confirmation, let us recall that it is based on $\operatorname{Pr}(E \mid H)=a /(a+b)$ and $\operatorname{Pr}(E \mid \neg H)=c /(c+d)$, so that the increase in $a$ and $d$ accompanied by a decrease in $b$ and $c$ widens the difference between $\operatorname{Pr}(E \mid H)$ and $\operatorname{Pr}(E \mid \neg H)$, making the first bigger and the latter smaller in value. Finally, for the general definition of confirmation, the increase in $a$ and $d$ accompanied by a decrease in $b$ and $c$ makes the value of $a d-b c$ larger, and thus reflecting a greater confirmation. Following only the situation of entailment, we would only demand an increase in $a$ and a decrease in $c$, which would erroneously limit the analysis.

Let us also observe that, in general, a confirmation measure monotonic in the Bayesian perspective or in the likelihoodist perspective does not satisfy the monotonicity M. To show this, let us take into account an example already given in [18] and consider the scenarios 5 and 6 described in Table 5.

Table 5. Contingency tables for scenario 5 and 6

\begin{tabular}{|c|c|c|c|}
\hline & $H_{5}$ & $\neg H_{5}$ & $\sum$ \\
\hline$E_{5}$ & $a_{5}=100$ & $c_{5}=0$ & $a_{5}+c_{5}=100$ \\
\hline$\neg E_{5}$ & $b_{5}=0$ & $d_{5}=1$ & $b_{5}+d_{5}=1$ \\
\hline$\sum$ & $a_{5}+b_{5}=100$ & $c_{5}+d_{5}=1$ & $|U|=101$ \\
\hline & & & \\
\hline & $H_{6}$ & $\neg H_{6}$ & $\sum$ \\
\hline$E_{6}$ & $a_{6}=101$ & $c_{6}=0$ & $a_{6}+c_{6}=101$ \\
\hline$\neg E_{6}$ & $b_{6}=0$ & $d_{6}=1$ & $b_{6}+d_{6}=1$ \\
\hline$\sum$ & $a_{6}+b_{6}=101$ & $c_{6}+d_{6}=1$ & $|U|=102$ \\
\hline
\end{tabular}


Measuring confirmation in scenario 5 and scenario 6 with confirmation measure $D(H, E)$ being monotonic in the Bayesian perspective, we get

$$
D\left(H_{5}, E_{5}\right)=1-\frac{100}{101} \leq 1-\frac{101}{102}=D\left(H_{6}, E_{6}\right),
$$

Thus, an increase in $a$ (from $a_{5}=100$ to $a_{6}=101$ ) results in a decrease of the confirmation measure $D(H, E)$ and therefore $D(H, E)$ does not satisfy the property of monotonicity $\mathrm{M}$.

Analogously, measuring confirmation in scenario 5 and scenario 6 with confirmation measure $M(H, E)$ being monotonic in the likelihoodist perspective, we get

$$
M\left(H_{5}, E_{5}\right)=1-\frac{100}{101} \leq 1-\frac{101}{102}=M\left(H_{6}, E_{6}\right),
$$

Again, an increase in $a$ (from $a_{5}=100$ to $a_{6}=101$ ) results in a decrease of the confirmation measure $M(H, E)$ and therefore $M(H, E)$ does not satisfy the property of monotonicity $\mathrm{M}$.

Thus, in general, measures monotonic with respect to left- and right-hand side probabilities of the Bayesian perspective (e.g., $D(H, E)$ ) or the likelihoodist perspective (e.g., $M(H, E)$ ) do not satisfy the property of monotonicity M.

Observe, however, that it is possible to have a confirmation measure monotonic in the Bayesian perspective, and satisfying monotonicity $\mathrm{M}$. This is the case of the following measure $Z(H, E)$ proposed by Crupi et al. [6]:

$$
Z(H, E)= \begin{cases}\frac{\operatorname{Pr}(H \mid E)-\operatorname{Pr}(H)}{1-\operatorname{Pr}(H)}=\frac{a d-b c}{(a+c)(c+d)} \quad \text { in case of confirmation } \\ \frac{\operatorname{Pr}(H \mid E)-\operatorname{Pr}(H)}{\operatorname{Pr}(H)}=\frac{a d-b c}{(a+c)(a+b)} \quad \text { in case of disconfirmation }\end{cases}
$$

Indeed, as one can verify by calculating partial derivatives, measure $Z(H, E)$ is strictly increasing with respect to $\operatorname{Pr}(H \mid E)$ and strictly decreasing with respect to $\operatorname{Pr}(H)$, and therefore it is monotonic in the Bayesian perspective. Moreover, $Z(H, E)$ respects monotonicity $\mathrm{M}$, as proved in [19].

It is also possible to have a confirmation measure monotonic in the likelihoodist perspective, and satisfying monotonicity $\mathrm{M}$. This is the case of the following measure $V(H, E)$ that can be obtained from $Z(H, E)$ by putting $V(H, E)=Z(E, H)$, that is:

$$
V(H, E)=\left\{\begin{array}{l}
\frac{\operatorname{Pr}(E \mid H)-\operatorname{Pr}(E)}{1-\operatorname{Pr}(E)}=\frac{a d-b c}{(a+b)(b+d)} \quad \text { in case of confirmation } \\
\frac{\operatorname{Pr}(E \mid H)-\operatorname{Pr}(E)}{\operatorname{Pr}(E)}=\frac{a d-b c}{(a+c)(a+b)} \quad \text { in case of disconfirmation }
\end{array}\right.
$$


One can verify by calculating partial derivatives, that $V(H, E)$ is strictly increasing with respect to $\operatorname{Pr}(E \mid H)$ and strictly decreasing with respect to $\operatorname{Pr}(E)$, and therefore it is monotonic in the likelihoodist perspective.

Moreover, $V(H, E)$ respects monotonicity $\mathrm{M}$ (see the proof of Observation 1 in the Appendix).

We can synthesize all this discussion in the following statement.

Proposition 1. Confirmation measures monotonic in the strong Bayesian or in the strong likelihoodist perspectives always satisfy the property of monotonicity $\mathrm{M}$, while confirmation measures monotonic in the Bayesian or in the likelihoodist perspectives may satisfy monotonicity M (i.e., there exist confirmation measures monotonic in the Bayesian or in the likelihoodist perspectives that satisfy monotonicity $\mathrm{M}$ as well as those that do not satisfy it).

\subsection{Weak Ex1 property}

The weak Ex 1 property introduced in [22] is a generalization of $\mathrm{Ex}_{1}$ property introduced by Crupi et al. in [6]. The original $\mathrm{Ex}_{1}$ property has been introduced as a requirement for confirmation being a generalization of logical entailment, however as such, it does not really work well in the context of rule evaluation by interestingness measures (see [22] and [16], followed by [17]). As we have argued earlier on, the entailment should rather be substituted with strategy inspired by strong Bayesian perspective. In this light, the substitution of original $\mathrm{Ex}_{1}$ property by weak $\mathrm{Ex}_{1}$ was proposed. It is recalled below.

On the basis of classical deductive logic Crupi et al. [6] constructed a function $v$ :

$$
v(H, E)=\left\{\begin{array}{l}
\text { the same positive value, denoted as } V, \\
\text { if } E \mid=H ; \\
\text { the same negative value, denoted as }-V, \\
\text { if } E \mid=\neg H ; \\
0, \text { otherwise. }
\end{array}\right.
$$

Function $v$ is such that it ascribes the same positive value (e.g., +1$)$ to any argument $(H, E)$ if and only if the rule's premise $E$ entails the conclusion $H$ (i.e., $E \mid=H$ ). If $E$ refutes $H$ (i.e., $E \mid=\neg H$ ) then the same value but of opposite 
sign (e.g., -1) is ascribed. On the other hand, when the premise is not conclusively confirmatory nor conclusively disconfirmatory for the conclusion, then function $v$ defaults to 0. Using function $v$, the original $\mathbf{E x}_{\mathbf{1}}$ property is defined as follows:

$$
\text { if } v\left(H_{1}, E_{1}\right)>v\left(H_{2}, E_{2}\right) \text {, then } c\left(H_{1}, E_{1}\right)>c\left(H_{2}, E_{2}\right)
$$

As we have observed in [22] such formulation of $\mathrm{Ex}_{1}$ implies that if a confirmation measure $c(H, E)$ reaches its maximal value, then there are no counterexamples to the rule, i.e., $E$ entails $H$, and if $c(H, E)$ reaches its minimal value, then there are no positive examples to the rule, i.e., $E$ entails $\neg H$. Nevertheless, it is still possible that $c=0$ and $c(H, E)$ does not obtain its maximal value, as well as, it is possible that $a=0$ and $c(H, E)$ does not get its minimal value.

One can observe that $\mathrm{Ex}_{1}$ is a desirable property for confirmation measures $c(H, E)$ interpreted as generalization of logical entailment $E \mid=H$. Moreover, it makes sense to consider disconfirmation as generalization of the entailment $\neg E \mid=H$, which leads to the following new property called $\mathbf{E x _ { 1 }}$ ':

$$
\text { if } v\left(H_{1}, \neg E_{1}\right)<v\left(H_{2}, \neg E_{2}\right) \text {, then } c\left(H_{1}, E_{1}\right)>c\left(H_{2}, E_{2}\right)
$$

In [22], another property was introduced, called weak $\mathbf{E x}_{1}$ :

$$
\begin{aligned}
& \text { if } v\left(H_{1}, E_{1}\right)>v\left(H_{2}, E_{2}\right) \text { and } v\left(H_{1}, \neg E_{1}\right)<v\left(H_{2}, \neg E_{2}\right), \\
& \text { then } c\left(H_{1}, E_{1}\right)>c\left(H_{2}, E_{2}\right) .
\end{aligned}
$$

Notice that the antecedent of property weak $\mathrm{Ex}_{1}$ is the conjunction of the antecedents of $\mathrm{Ex}_{1}$ and $\mathrm{Ex}_{1}$ ', while the consequent is the same for all three properties. Property $\mathrm{Ex}_{1}$ says that $c(H, E)$ cannot attain its maximum (minimum) value unless $E \mid=H(E \mid=\neg H)$, i.e., $c=0 \quad(a=0)$. Analogously, property $\mathrm{Ex}_{1}$ ' says that $c(H, E)$ cannot attain its maximum (minimum) value unless $\neg E \mid=\neg H(\neg E \mid=H)$, i.e., $b=0(d=0)$. Interpretation of property weak $\mathrm{Ex}_{1}$ is formulated in the following theorem.

Theorem 2. Under weak $\mathrm{Ex}_{1}$, confirmation measure $c(H, E)$ cannot attain its maximum value unless $E \mid=H$ or $\neg E \mid=\neg H$, i.e., $c=0$ or $b=0$. Confirmation measure $c(H, E)$ satisfying weak $\mathrm{Ex}_{1}$ cannot attain its minimum value unless $E \mid=\neg H$ or $\neg E \mid=H$, i.e., $a=0$ or $d=0$.

It should be noted that the above formulation and interpretation of weak $\mathrm{Ex}_{1}$ corrects those given without proof in [22] and [23]. 
Notice moreover that a confirmation measure satisfying $\mathrm{Ex}_{1}$ or $\mathrm{Ex}_{1}$ ' satisfies also weak $\mathrm{Ex}_{1}$. Indeed, for confirmation measure $c(H, E)$ satisfying $\mathrm{Ex}_{1}$, condition $v\left(H_{1}, E_{1}\right)>v\left(H_{2}, E_{2}\right)$ is sufficient for $c\left(H_{1}, E_{1}\right)>c\left(H_{2}, E_{2}\right)$, which continues to hold if, moreover, $v\left(H_{1}, \neg E_{1}\right)<v\left(H_{2}, \neg E_{2}\right)$, which amounts to weak $E_{1}$. Analogous proof holds for Ex ${ }_{1}$. One can observe, however, that the opposite claim is not true. Take, for example, confirmation measure [5]:

$$
S(H, E)=\operatorname{Pr}(H \mid E)-\operatorname{Pr}(H \mid \neg E)=\frac{a}{a+c}-\frac{b}{b+d} .
$$

Obviously, the maximum value of $S(H, E)$ is 1 , which is attained when $c=0$ and $b=0$ (measure $S(H, E)$ satisfies weak $\mathrm{Ex}_{1}$ ). When $c=0$, but $b>0$, then $S(H, E)<1$, thus $\mathrm{Ex}_{1}$ does not hold. Analogously, when $b=0$, but $c>0$, then also $S(H, E)<1$, thus $\mathrm{Ex}_{1}$ ' does not hold as well.

Observe that one can also consider the following likelihoodist counterpart of weak $\mathrm{Ex}_{1}$ property, that we call weak $\mathbf{L}-\mathbf{E x}_{1}$ property:

$$
\begin{aligned}
& \text { if } v\left(E_{1}, H_{1}\right)>v\left(E_{2}, H_{2}\right) \text { and } v\left(E_{1}, \neg H_{1}\right)<v\left(E_{2}, \neg H_{2}\right), \\
& \text { then } c\left(H_{1}, E_{1}\right)>c\left(H_{2}, E_{2}\right) .
\end{aligned}
$$

The following result is the counterpart of Theorem 2 with respect to weak $\mathrm{L}-\mathrm{Ex}_{1}$ property.

Theorem 3. Under weak L-Ex 1 , confirmation measure $c(H, E)$ cannot attain its maximum value unless $H \mid=E$ or $\neg H \mid=\neg E$, i.e., $b=0$ or $c=0$. Confirmation measure $c(H, E)$ satisfying weak L-Ex $x_{1}$ cannot attain its minimum value unless $H \mid=\neg E$ or $\neg H \mid=E$, i.e., $a=0$ or $d=0$.

The following result shows the intimate relations between weak $\mathrm{Ex}_{1}$ property and confirmation measures monotonic in the strong Bayesian perspective on one hand and weak L-Ex 1 property and confirmation measures monotonic in the strong likelihoodist perspective on the other hand.

Theorem 4. Confirmation measures strictly monotonic in the strong Bayesian perspective satisfy weak $\mathrm{Ex}_{1}$ property. Confirmation measures strictly monotonic in the strong likelihoodist perspective satisfy weak L-Ex 1 property.

\subsection{Weak $L$ property}

Closely related to $\mathrm{Ex}_{1}, \mathrm{Ex}_{1}$ ' and weak $\mathrm{Ex}_{1}$ properties are properties $\mathrm{L}$ (also known as logicality) [6], [16], and weak L [22], [23], and maximality/minimality [17]. The original L property (similarly to $\mathrm{Ex}_{1}$ 
property) was inspired by understanding confirmation as a generalization of logical entailment. Precisely, property $\mathbf{L}$ says that:

- if $E \mid=H$ (equivalently, if $c=0$ ), then $c(H, E)$ attains its maximum value, and

- if $E \mid=\neg H$ (equivalently, if $a=0$ ), then $c(H, E)$ attains its minimum value.

A complementary property to $\mathrm{L}$ would be the following new property L':

- if $\neg E \mid=\neg H$ (equivalently, if $b=0$ ), then $c(E, H)$ attains its maximum value, and

- if $\neg E \mid=H$ (equivalently, if $d=0$ ), then $c(E, H)$ attains its minimum value.

In [22], another property was introduced, called weak $\mathbf{L}$ assuring that:

- if $E \mid=H$ and $\neg E \mid=\neg H$, or equivalently when $c=b=0$, then $c(H, E)$ is maximal and

- if $E \mid=\neg H$ and $\neg E \mid=H$, or equivalently when $a=d=0$, then $c(H, E)$ is minimal.

Notice that the antecedent of property weak $\mathrm{L}$ is the conjunction of the antecedents of L and L', while the consequent is the same for all three properties.

Observe that, the formulation of weak L property is closely related to the formulation of strong Bayesian confirmation as it associates the maximum value to $c(E, H)$ when $\operatorname{Pr}(H \mid E)=a /(a+c)=1$ and $\operatorname{Pr}(H \mid \neg E)=b /(b+d)=0$, as well as it associates the minimum value to $c(E, H)$ when $\operatorname{Pr}(H \mid E)=a /(a+c)=0$ and $\operatorname{Pr}(H \mid \neg E)=b /(b+d)=1$.

Close to weak $\mathrm{Ex}_{1}$ and weak $\mathrm{L}$ properties is also the maximality/minimality requirement proposed by Glass in [16], [17], stating that:

- $c(H, E)$ should be maximal if and only if $b=c=0$ and

- $c(H, E)$ should be minimal if and only if $a=d=0$,

provided that $c(H, E)$ is defined.

Theorem 5. Confirmation measures monotonic in the strong Bayesian perspective and measures monotonic in the strong likelihoodist perspective satisfy weak L property. Confirmation measures strictly monotonic in the strong Bayesian perspective, as well as confirmation measures strictly monotonic in the strong likelihoodist perspective, satisfy maximality/minimality. 


\subsection{Symmetry properties}

Among widely considered properties for confirmation measures there is also a group of symmetry properties. Different authors are however not unanimous as to which particular symmetries are desirable.

Inspired by the work of Carnap [4], Eells and Fitelson have investigated in [7] the following four properties of symmetry:

- inversion (commutativity) symmetry $I S: c(H, E)=c(E, H)$,

- evidence symmetry $E S: c(H, E)=-c(H, \neg E)$,

- hypothesis symmetry $H S: c(H, E)=-c(\neg H, E)$,

- evidence-hypothesis (total) symmetry EHS: $\quad c(H, E)=c(\neg H, \neg E)$. Eells and Fitelson concluded in [7] that, in fact, only hypothesis symmetry $H S$ is a desirable property, while evidence symmetry $E S$, commutativity symmetry $I S$ and total symmetry $E H S$ are not. Such symmetry requirements are also seen as favorable by Glass in [16].

In their work [6], Crupi et at. have revised the symmetries considered by Eells and Fitelson in [7] and proposed to widen the analysis of symmetry properties. As a result, they investigated 7 symmetries (their analysis was conducted first for the situation of confirmation and then for that of disconfirmation) being all combinations obtained by negation and/or inversion of the premise $E$ and the hypothesis $H$ :

- $E S: c(H, E)=-c(H, \neg E)$,

- $H S: c(H, E)=-c(\neg H, E)$,

- $E I S: c(H, E)=-c(\neg E, H)$,

- $H I S: c(H, E)=-c(E, \neg H)$,

- IS: $c(H, E)=c(E, H)$,

- $E H S: c(H, E)=c(\neg H, \neg E)$,

- EHIS: $c(H, E)=c(\neg E, \neg H)$.

The results obtained by Crupi et al. point that in case of confirmation only $H S, H I S$ and EHIS are the desirable properties. In case of disconfirmation, they favor $H S, E I S$ and $I S$ properties, finding all other symmetries as unattractive. The results obtained by Crupi et al. concur with Eells and Fitelson in case of confirmation.

The argumentation presented both in the work of Eells and Fitelson, and of Crupi et al. is based on understanding Bayesian confirmation as a generalization of logical entailment. The counterexample they use in [7] to rule out evidence symmetry is based on a conclusively confirmatory rule: if $a$ randomly drawn card is the seven of spades, then the card is black. They remark that knowing that we have drawn the seven of spades is a much 
stronger evidence for confirming the conclusion than the negated evidence, i.e., knowing that the drawn card is not the seven of spades, is for disconfirming the same conclusion. Thus, according to Eells and Fitelson, the equality in evidence symmetry is found unattractive, i.e., for some situation $c(H, E) \neq-c(H, \neg E))$.

However, since boiling the considerations down to entailment or refutation is, in our opinion, unwarranted in case of confirmation measures, we reconsidered the symmetry properties in the light of strong Bayesian confirmation [23]. The analysis brought us to the conclusion that there is no need to consider the cases of confirmation and disconfirmation separately like Crupi et al. did., and that only $H S, E S$ and $E H S$ are desirable symmetry properties.

First of all, let us observe that in the strong Bayesian perspective both confirmation and disconfirmation are based on the consideration of $\operatorname{Pr}(H \mid E)$ and $\operatorname{Pr}(H \mid \neg E)$. The highest confirmation is obtained when $\operatorname{Pr}(H \mid \neg E)=0$ and $\operatorname{Pr}(H \mid E)=1$, whereas the highest disconfirmation, occurs when $\operatorname{Pr}(H \mid \neg E)=1$ and $\operatorname{Pr}(H \mid E)=0$. Thus, we argued in [23] to consider the symmetry properties together for cases of confirmation and disconfirmation as they both consider passing from $\operatorname{Pr}(H \mid \neg E)$ to $\operatorname{Pr}(H \mid E)$.

To recall our argumentation for or against particular symmetry properties [23], let us use an exemplary scenario 3 from Table 4, where the values from contingency table of $E_{3}$ and $H_{3}$ are: $a_{3}=100, b_{3}=99, c_{3}=0, d_{3}=1,|U|=200$ and apply it for showing that evidence symmetry is desirable. Let us observe, that for $c\left(H_{3}, E_{3}\right)$ we have that $\operatorname{Pr}\left(H_{3} \mid \neg E_{3}\right)=0.99$ and $\operatorname{Pr}\left(H_{3} \mid E_{3}\right)=1$, which gives us a $1 \%$ increase of confirmation. On the other hand, for $c\left(H_{3}, \neg E_{3}\right)$ we get $\operatorname{Pr}\left(H_{3} \mid E_{3}\right)=1$ and $\operatorname{Pr}\left(H_{3} \mid \neg E_{3}\right)=0.99$, which results in $1 \%$ decrease of confirmation. Thus, clearly the confirmation of a rule $E_{3} \rightarrow H_{3}$ should be of the same value but of the opposite sign as the confirmation of a $\neg E_{3} \rightarrow H_{3}$ rule. Therefore, we can conclude that $\left.c\left(H_{3}, E_{3}\right)=-c\left(H_{3}, \neg E_{3}\right)\right)$, i.e., evidence symmetry, is desirable.

The considerations for other symmetry properties can be conducted analogously. Our results, i.e., seeing $H S, E S$ and $E H S$ as desirable, differ from what Eells and Fitelson [7], and Crupi et al. [6] advocated for because they treat the entailment of the conclusion by the premise as the maximal confirmation, whereas we consider the increase of confirmation when passing from the absence of the premise $(\neg E)$ to its presence $(E)$.

Now, let us present and discuss possible behaviors of confirmation measures monotonic in the four perspectives, with respect to the above symmetries. 
Theorem 6. Consider a confirmation measure $c_{s m B}(H, E)$ strictly monotonic in the Bayesian perspective. The following statements hold:

1) there are no confirmation measures $c_{s m B}(H, E)$ that satisfy $E S$;

2) there exist confirmation measures $c_{s m B}(H, E)$ that satisfy $H S$;

3) there are no confirmation measures $c_{s m B}(H, E)$ that satisfy $E I S$;

4) there are no confirmation measures $c_{s m B}(H, E)$ that satisfy $H I S$;

5) there exist confirmation measures $c_{s m B}(H, E)$ that satisfy $I S$;

6) there are no confirmation measures $c_{s m B}(H, E)$ that satisfy $E H S$;

7) there exist confirmation measures $c_{s m B}(H, E)$ that satisfy EHIS.

Moreover, if $c_{s m B}(H, E)$ satisfies one among $H S, I S$ and $E H I S$, it cannot satisfy any of the remaining two symmetry properties. Finally, there are confirmation measures $c_{s m B}(H, E)$ that do not satisfy any symmetry property.

Observe that on the basis of Theorem 6 we can classify confirmation measures strictly monotonic in the Bayesian perspective in four exhaustive and mutually exclusive classes:

- confirmation measures $c_{s m B}(H, E)$ satisfying symmetry property $H S$, such as

$$
c_{s m B}(H, E)=\operatorname{Pr}(H \mid E)-\operatorname{Pr}(H),
$$

- confirmation measures $c_{s m B}(H, E)$ satisfying symmetry property $I S$, such as

$$
c_{s m B}(H, E)=\frac{\operatorname{Pr}(H \mid E)-\operatorname{Pr}(H)}{\operatorname{Pr}(H \mid E)+\operatorname{Pr}(H)},
$$

- confirmation measures $c_{s m B}(H, E)$ satisfying symmetry property $E H I S$, such as

$$
c_{s m B}(H, E)=\frac{\operatorname{Pr}(H \mid E)-\operatorname{Pr}(H)}{2-\operatorname{Pr}(H \mid E)-\operatorname{Pr}(H)},
$$

- confirmation measures $c_{s m B}(H, E)$ satisfying no symmetry property, such as

$$
c_{s m B}(H, E)=\sqrt{\operatorname{Pr}(H \mid E)}-\sqrt{\operatorname{Pr}(H)} .
$$

Theorem 7. Consider a confirmation measure $c_{s m S B}(H, E)$ strictly monotonic in the strong Bayesian perspective. For any symmetry property there are confirmation measures $c_{s m S B}(H, E)$ satisfying it. Moreover, there are confirmation measures $c_{s m S B}(H, E)$ that satisfy all symmetry properties.

Continuing our discussion on the relationship between measures monotonic in different perspectives and different symmetries, let us now introduce some new definitions and lemmas that, besides being interesting per se, are useful in proving the following theorems: Theorem 9 and Theorem 10. 
Definition 2. Given a confirmation measure $c(H, E)$, we say that:

- $c(H, E)$ is monotonic in the converse Bayesian perspective if there exists $f:[0,1] \times[0,1] \rightarrow \Re$ non-decreasing with the first argument and non-increasing with the second argument, with $f(x, x)=0$ for all $x \in[0,1]$, such that $c(H, E)=-f(\operatorname{Pr}(H \mid \neg E), \operatorname{Pr}(H))$,

- $c(H, E)$ is monotonic in the converse likelihoodist perspective if there exists $f:[0,1] \times[0,1] \rightarrow \Re$ non-decreasing with the first argument and non-increasing with the second argument with $f(x, x)=0$ for all $x \in[0,1]$, such that $c(H, E)=-f(\operatorname{Pr}(E \mid \neg H), \operatorname{Pr}(E))$.

Let us observe that the converse Bayesian perspective and the converse likelihoodist perspective express that $E$ confirms $H$ since

- $\operatorname{Pr}(H)>\operatorname{Pr}(H \mid \neg E)$ and

- $\operatorname{Pr}(E)>\operatorname{Pr}(E \mid \neg H)$, respectively,

are logically equivalent (as the other above considered perspectives of confirmation) to $\operatorname{Pr}(H \mid E)>\operatorname{Pr}(H)$.

Indeed, since $\operatorname{Pr}(H \mid E)>\operatorname{Pr}(H)$ is logically equivalent to $\operatorname{Pr}(H \mid E)>\operatorname{Pr}(H \mid \neg E)$, we get

$\operatorname{Pr}(H)=\operatorname{Pr}(H \mid E) \operatorname{Pr}(E)+\operatorname{Pr}(H \mid \neg E)(1-\operatorname{Pr}(E))$

$>$

$\operatorname{Pr}(H \mid \neg E) \operatorname{Pr}(E)+\operatorname{Pr}(H \mid \neg E)(1-\operatorname{Pr}(E))=$ $\operatorname{Pr}(H \mid \neg E)$.

Analogously, since $\operatorname{Pr}(H \mid E)>\operatorname{Pr}(H)$ is equivalent to $\operatorname{Pr}(E \mid H)>\operatorname{Pr}(E \mid \neg H)$, we get

$$
\begin{aligned}
& \operatorname{Pr}(E)=\operatorname{Pr}(E \mid H) \operatorname{Pr}(H)+\operatorname{Pr}(E \mid \neg H)(1-\operatorname{Pr}(H)) \\
& > \\
& \operatorname{Pr}(E \mid \neg H) \operatorname{Pr}(H)+\operatorname{Pr}(E \mid \neg H)(1-\operatorname{Pr}(H))=\operatorname{Pr}(E \mid \neg H) .
\end{aligned}
$$

To provide an interpretation for the converse Bayesian perspective and the converse likelihoodist perspectives of confirmation let us use again the example, in which the premise $E$ is the evidence that a patient suffered from a fever and the conclusion $H$ reflects that the patient had a flu. Then:

- in case of converse Bayesian perspective of confirmation, if flu is more probable without knowing whether the fever occurred or not than knowing that there is not fever, then fever confirms flu,

- in case of converse likelihoodist perspective of confirmation, if fever is more probable without knowing whether the flu occurred or not than knowing that there is not flu, then fever confirms flu. 
Theorem 8. A confirmation measure $c(H, E)=f\left(\operatorname{Pr}_{\mathrm{L}}, \operatorname{Pr}_{\mathrm{R}}\right)$ being strictly monotonic in the converse Bayesian or the converse likelihoodist perspective is not strictly monotonic in the other perspectives.

Given a confirmation measure $c(H, E)$, let us observe that applying any of the transformations related to the symmetries $X \in\{E S, H S, E I S, H I S, I S, E H S, E H I S\}$ we get another confirmation measure $c^{X}(H, E)$. For example, considering confirmation measure

$$
D(H, E)=\operatorname{Pr}(H \mid E)-\operatorname{Pr}(H)
$$

and applying the transformation related to the $I S$ symmetry we get

$$
D^{E S}(H, E)=\operatorname{Pr}(E \mid H)-\operatorname{Pr}(E)=M(H, E) .
$$

Lemma 1. If $c(H, E)$ is a confirmation measure monotonic in the perspective $\mathrm{P}$, then $c^{X}(H, E)$ is monotonic in the perspective showed in Table 6 , where $\mathrm{P} \in\{$ Bayesian $(B)$, strong Bayesian $(S B)$, likelihoodist $(L)$, strong likelihoodist $(S L)$, converse Bayesian $(C B)$, converse likelihoodist $(C L)\}$, $X \in\{E S, H S, E I S, H I S, I S, E H S, E H I S\}$.

Table 6. Transformation of perspectives of confirmation measures after negation and/or inversion of evidence $E$ and hypothesis $H$

\begin{tabular}{|l|l|l|l|l|l|l|}
\hline $\boldsymbol{X} \backslash \mathbf{P}$ & $\boldsymbol{B}$ & $\boldsymbol{S B}$ & $\boldsymbol{L}$ & $\boldsymbol{S L}$ & $\boldsymbol{C B}$ & $\boldsymbol{C L}$ \\
\hline $\boldsymbol{E S}$ & $C B$ & $S B$ & $L$ & $S L$ & $B$ & $C L$ \\
\hline $\boldsymbol{H S}$ & $B$ & $S B$ & $C L$ & $S L$ & $C B$ & $L$ \\
\hline $\boldsymbol{E I S}$ & $L$ & $S L$ & $C B$ & $S B$ & $C L$ & $B$ \\
\hline $\boldsymbol{H I S}$ & $C L$ & $S L$ & $B$ & $S B$ & $L$ & $C B$ \\
\hline $\boldsymbol{I S}$ & $L$ & $S L$ & $B$ & $S B$ & $C L$ & $C B$ \\
\hline $\boldsymbol{E H S}$ & $C B$ & $S B$ & $C L$ & $S L$ & $B$ & $L$ \\
\hline $\boldsymbol{E H I S}$ & $C L$ & $S L$ & $C B$ & $S B$ & $L$ & $B$ \\
\hline
\end{tabular}

Observe that Lemma 1 says that the six perspectives of confirmation we have considered from the viewpoint of monotonicity are exhaustive, because no other new perspective of confirmation measures can be build up by transformations of confirmation measures related to symmetry properties.

Lemma 2. For any transformation $c^{X}(H, E)$ of $c(H, E)$, where $X \in\{E S, H S, E I S, H I S, I S, E H S, E H I S\}$, there exists an inverse transformation $X^{-1}$ such that $c^{X X^{-1}}(H, E)=c^{X^{-1} X}(H, E)=c(H, E)$.

More precisely, we have:

- $E S^{-1}=E S$,

- $H S^{-1}=H S$, 
- $E I S^{-1}=H I S$,

- $H I S^{-1}=E I S$,

- $I S^{-1}=I S$,

- $E H S^{-1}=E H S$,

- $E H I S^{-1}=E H I S$.

Lemma 3. Given confirmation measures $c(H, E)$ and $c_{1}(H, E)$ and $X, Y \in\{E S, H S, E I S, H I S, I S, E H S, E H I S\}, \quad$ such that $c(H, E)=c_{1}^{X}(H, E)$, confirmation measure $c_{1}(H, E)$ satisfies symmetry property $Y$, that is $c_{1}(H, E)=$ $c_{1}^{Y}(H, E)$, if and only if confirmation measure $c(H, E)$ satisfies property $X Y X^{-1}$, that is $c(H, E)=c^{X Y X^{-1}}(H, E)$.

Using Lemma 3 and the results of Theorem 6 concerning confirmation measures strictly monotonic in the Bayesian perspective, we can easily find analogous results for confirmation measures strictly monotonic in the likelihoodist, converse Bayesian and converse likelihoodist perspectives. Moreover, the results of Theorem 7 can be extended from confirmation measures strictly monotonic in the strong Bayesian perspective to confirmation measures strictly monotonic in the strong likelihoodist perspective.

Theorem 9. Consider a confirmation measure

- $c_{s m L}(H, E)$ strictly monotonic in the likelihoodist perspective, i.e., being strictly increasing with respect to $\operatorname{Pr}(E \mid H)$ and strictly decreasing with respect to $\operatorname{Pr}(E)$,

- $c_{s m C B}(H, E)$ strictly monotonic in the converse Bayesian perspective, i.e., being strictly decreasing with respect to $\operatorname{Pr}(H \mid \neg E)$ and strictly increasing with respect to $\operatorname{Pr}(H)$,

- $c_{s m C L}(H, E)$ strictly monotonic in the converse likelihoodist perspective, i.e., being strictly decreasing with respect to $\operatorname{Pr}(E \mid \neg H)$ and strictly increasing with respect to $\operatorname{Pr}(E)$.

We have that:

1) there exist confirmation measures $c_{s m L}(H, E)$ and $c_{s m C L}(H, E)$ that satisfy $E S$, while there are no confirmation measures $c_{s m C B}(H, E)$ that satisfy $E S$,

2) there exist confirmation measures $c_{s m C B}(H, E)$ that satisfy $H S$, while there are no confirmation measures $c_{s m L}(H, E)$ and $c_{s m C L}(H, E)$ that satisfy $H S$;

3) there are no confirmation measures $c_{s m L}(H, E), \quad c_{s m C B}(H, E)$ and $c_{s m C L}(H, E)$ that satisfy $E I S$; 
4) there are no confirmation measures $c_{s m L}(H, E), \quad c_{s m C B}(H, E)$ and $c_{s m C L}(H, E)$ that satisfy $H I S$;

5) there exist confirmation measures $c_{s m L}(H, E), \quad c_{s m C B}(H, E)$ and $c_{s m C L}(H, E)$ that satisfy $I S$;

6) there are no confirmation measures $c_{s m L}(H, E), \quad c_{s m C B}(H, E)$ and $c_{s m C L}(H, E)$ that satisfy $E H S$;

7) there exist confirmation measures $c_{s m L}(H, E), \quad c_{s m C B}(H, E)$ and $c_{s m C L}(H, E)$ that satisfy EHIS.

Moreover, if $c_{s m C B}(H, E)\left(c_{s m L}(H, E)\right.$ or $\left.c_{s m C L}(H, E)\right)$ satisfies one among $H S$, $I S$ and EHIS (one among ES, IS and EHIS), it cannot satisfy any of the remaining two symmetry properties. Finally, there are confirmation measures $c_{s m L}(H, E), c_{s m C B}(H, E)$ and $c_{s m C L}(H, E)$ that do not satisfy any symmetry property.

Observe that on the basis of Theorem 9 we can classify confirmation measures $c_{s m L}(H, E)$ strictly monotonic in the likelihoodist perspective in four exhaustive and mutually exclusive classes:

- confirmation measures $c_{s m L}(H, E)$ satisfying symmetry property $E S$, such as

$$
c_{s m L}(H, E)=\operatorname{Pr}(E \mid H)-\operatorname{Pr}(E),
$$

- confirmation measures $c_{s m L}(H, E)$ satisfying symmetry property $I S$, such as

$$
c_{s m L}(H, E)=\frac{\operatorname{Pr}(E \mid H)-\operatorname{Pr}(E)}{\operatorname{Pr}(E \mid H)+\operatorname{Pr}(E)},
$$

- confirmation measures $c_{s m L}(H, E)$ satisfying symmetry property $E H I S$, such as

$$
c_{s m L}(H, E)=\frac{\operatorname{Pr}(E \mid H)-\operatorname{Pr}(E)}{2-\operatorname{Pr}(E \mid H)-\operatorname{Pr}(E)},
$$

- confirmation measures $c_{s m L}(H, E)$ satisfying no symmetry property, such as

$$
c_{s m L}(H, E)=\sqrt{\operatorname{Pr}(E \mid H)}-\sqrt{\operatorname{Pr}(E)} .
$$

Moreover, on the basis of Theorem 9, we can classify confirmation measures $c_{s m C B}(H, E)$ strictly monotonic in the converse Bayesian perspective in four exhaustive and mutually exclusive classes:

- confirmation measures $c_{s m C B}(H, E)$ satisfying symmetry property $H S$, such as

$$
c_{s m C B}(H, E)=\operatorname{Pr}(H)-\operatorname{Pr}(H \mid \neg E),
$$

- confirmation measures $c_{s m C B}(H, E)$ satisfying symmetry property $I S$, such as 


$$
c_{s m C B}(H, E)=\frac{\operatorname{Pr}(H)-\operatorname{Pr}(H \mid \neg E)}{\operatorname{Pr}(H)+\operatorname{Pr}(H \mid \neg E)},
$$

- confirmation measures $c_{s m C B}(H, E)$ satisfying symmetry property EHIS, such as

$$
c_{s m C B}(H, E)=\frac{\operatorname{Pr}(H)-\operatorname{Pr}(H \mid \neg E)}{2-\operatorname{Pr}(H)-\operatorname{Pr}(H \mid \neg E)},
$$

- confirmation measures $c_{s m C B}(H, E)$ satisfying no symmetry property, such as

$$
c_{s m C B}(H, E)=\sqrt{\operatorname{Pr}(H)}-\sqrt{\operatorname{Pr}(H \mid \neg E)} .
$$

Finally, on the basis of Theorem 9, we can classify also confirmation measures $c_{s m C L}(H, E)$ strictly monotonic in the converse likelihoodist perspective in four exhaustive and mutually exclusive classes:

- confirmation measures $c_{s m C L}(H, E)$ satisfying symmetry property $E S$, such as

$$
c_{s m C L}(H, E)=\operatorname{Pr}(E)-\operatorname{Pr}(E \mid \neg H),
$$

- confirmation measures $c_{s m C L}(H, E)$ satisfying symmetry property $I S$, such as

$$
c_{s m C L}(H, E)=\frac{\operatorname{Pr}(E)-\operatorname{Pr}(E \mid \neg H)}{\operatorname{Pr}(E)+\operatorname{Pr}(E \mid \neg H)},
$$

- confirmation measures $c_{s m C L}(H, E)$ satisfying symmetry property EHIS, such as

$$
c_{s m C L}(H, E)=\frac{\operatorname{Pr}(E)-\operatorname{Pr}(E \mid \neg H)}{2-\operatorname{Pr}(E)-\operatorname{Pr}(E \mid \neg H)},
$$

- confirmation measures $c_{s m C L}(H, E)$ satisfying no symmetry property, such as

$$
c_{s m C L}(H, E)=\sqrt{\operatorname{Pr}(E)}-\sqrt{\operatorname{Pr}(E \mid \neg H)} .
$$

Theorem 10. Consider a confirmation measure $c_{s m S L}(H, E)$ strictly monotonic in the strong likelihoodist perspective, i.e., being strictly increasing with respect to $\operatorname{Pr}(E \mid H)$ and strictly decreasing with respect to $\operatorname{Pr}(\neg E \mid H)$. For any symmetry property there are confirmation measures $c_{s m S L}(H, E)$ satisfying it. Moreover, there are confirmation measures $c_{s m S L}(H, E)$ that satisfy all symmetry properties.

Let us observe that the hypothesis of strict monotonicity in the above theorems cannot be removed, because, indeed, there exist confirmation 
measures that are monotonic, but not strictly monotonic, in all the perspectives and satisfy all the symmetry properties. This is the case of confirmation measure $c_{U}(H, E)$ presented below, which is "universally" monotonic in all the six perspectives:

$$
c_{U}(H, E)=\left\{\begin{array}{l}
1 \quad \text { if } a d-b c>0, \\
0 \quad \text { if } a d-b c=0, \\
-1 \text { if } a d-b c<0 .
\end{array}\right.
$$

It is easy to see that one can write $c_{U}(H, E)=f\left(\operatorname{Pr}_{\mathrm{L}}, \operatorname{Pr}_{\mathrm{R}}\right)$ in terms of left- and right-hand side probabilities $\operatorname{Pr}_{L}$ and $\operatorname{Pr}_{R}$ of any of the six perspectives as follows:

$$
c_{U}(H, E)= \begin{cases}1 & \text { if } \mathrm{Pr}_{\mathrm{L}}>\mathrm{Pr}_{\mathrm{R}}, \\ 0 & \text { if } \mathrm{Pr}_{\mathrm{L}}=\mathrm{Pr}_{\mathrm{R}}, \\ -1 & \text { if } \mathrm{Pr}_{\mathrm{L}}<\mathrm{Pr}_{\mathrm{R}} .\end{cases}
$$

\section{Selected measures of confirmation}

Measures that possess the property of confirmation defined as Bayesian confirmation, strong Bayesian confirmation, likelihoodist confirmation, strong likelihoodist confirmation, converse Bayesian confirmation or converse likelihoodist confirmation are referred to as confirmation measures or measures of confirmation. Sometimes an adjective comes into the denotation, e.g., Bayesian confirmation measures.

Due to the logical equivalence of all the analyzed perspectives of confirmation we can conclude that a measure satisfying the Bayesian confirmation must also satisfy strong Bayesian confirmation, likelihoodist confirmation, etc., as long as we exclude undefined values. It is thus legitimate to call such measures simply measures of confirmation (or confirmation measures).

Such measures quantify the degree to which the premise $E$ provides "support for or against" the conclusion $H$ [12], the degree to which $E$ confirms/disconfirms $H$. By using confirmation measures in the rule evaluation process, we aim at limiting the set of rules proposed to the user [40]. Let us observe, that the constraints put on a measure by any of the 
perspectives of confirmation are that a measure assigns positive values in the situation when confirmation occurs, negative values in case of disconfirmation, and zero otherwise. In consequence of that many alternative, non-equivalent measures of confirmation have been defined. Here we select for further analysis the normalized measures shown in Table 7.

Table 7. Selected measures of confirmation

\begin{tabular}{|l|l|}
\hline$D(H, E)=\operatorname{Pr}(H \mid E)-\operatorname{Pr}(H)=\frac{a}{a+c}-\frac{a+b}{|U|}$ & {$[4]$} \\
\hline$M(H, E)=\operatorname{Pr}(E \mid H)-\operatorname{Pr}(E)=\frac{a}{a+b}-\frac{a+c}{|U|}$ & {$[31]$} \\
\hline$S(H, E)=\operatorname{Pr}(H \mid E)-\operatorname{Pr}(H \mid \neg E)=\frac{a}{a+c}-\frac{b}{b+d}$ & {$[5]$} \\
\hline$N(H, E)=\operatorname{Pr}(E \mid H)-\operatorname{Pr}(E \mid \neg H)=\frac{a}{a+b}-\frac{c}{c+d}$ & {$[33]$} \\
\hline$C(H, E)=\operatorname{Pr}(E \wedge H)-\operatorname{Pr}(E) \operatorname{Pr}(H)=\frac{a}{|U|}-\frac{(a+c)(a+b)}{|U|^{2}}$ & {$[4]$} \\
\hline$F(H, E)=\frac{\operatorname{Pr}(E \mid H)-\operatorname{Pr}(E \mid \neg H)}{\operatorname{Pr}(E \mid H)+\operatorname{Pr}(E \mid \neg H)}=\frac{a d-b c}{a d+b c+2 a c}$ & {$[25]$} \\
\hline$F^{\prime}(H, E)=\frac{\operatorname{Pr}(H \mid E)-\operatorname{Pr}(H \mid \neg E)}{\operatorname{Pr}(H \mid E)+\operatorname{Pr}(H \mid \neg E)}=\frac{a d-b c}{a d+b c+2 a b}$ & {$[6]$} \\
\hline$Z(H, E)=\left\{\begin{array}{l}\frac{\operatorname{Pr}(H \mid E)-\operatorname{Pr}(H)}{1-\operatorname{Pr}(H)}=\frac{a d-b c}{(a+c)(c+d)} \text { in case of confirmation } \\
\hline \frac{\operatorname{Pr}(H \mid E)-\operatorname{Pr}(H)}{\operatorname{Pr}(H)}=\frac{a d-b c}{(a+c)(a+b)} \text { in case of disconfirmation }\end{array}\right.$ & {$\left[\begin{array}{l}\operatorname{Pr}(H)-\operatorname{Pr}(H \mid \neg E) \\
\operatorname{Pr}(H) \\
\frac{\operatorname{Pr}(H)-\operatorname{Pr}(H \mid \neg E)}{1-\operatorname{Pr}(H)}=\frac{a d-b c}{(a+b)(b+d)} \text { in case of confirmation } \\
A(H, E)(c+d)\end{array}\right.$}
\end{tabular}




\begin{tabular}{|c|c|}
\hline $\begin{array}{l}c_{1}(H, E)=\left\{\begin{array}{c}\alpha+\beta A(H, E) \text { in case of confirmation when } \mathrm{c}=0 \\
\alpha Z(H, E) \text { in case of confirmation when } \mathrm{c}>0 \\
\alpha Z(H, E) \text { in case of disconfirmation when } \mathrm{a}>0 \\
-\alpha+\beta A(H, E) \text { in case of disconfirmation when } \mathrm{a}=0\end{array}\right. \\
\text { where } \alpha \geq 0, \beta \geq 0 \text { and } \alpha+\beta=1\end{array}$ & [22] \\
\hline $\begin{array}{l}c_{2}(H, E)=\left\{\begin{array}{c}\alpha+\beta Z(H, E) \text { in case of confirmation when } \mathrm{b}=0 \\
\alpha A(H, E) \text { in case of confirmation when } \mathrm{b}>0 \\
\alpha A(H, E) \text { in case of disconfirmation when } \mathrm{d}>0\end{array}\right. \\
\text { where } \alpha \geq 0, \beta \geq 0 \text { and } \alpha+\beta=1\end{array}$ & [22] \\
\hline$c_{3}(H, E)=\left\{\begin{array}{c}A(H, E) Z(H, E) \text { in case of confirmation } \\
-A(H, E) Z(H, E) \text { in case of disconfirmation }\end{array}\right.$ & [22] \\
\hline$c_{4}(H, E)=\left\{\begin{array}{c}\min (A(H, E), Z(H, E)) \text { in case of confirmation } \\
\max (A(H, E), Z(H, E)) \text { in case of disconfirmation }\end{array}\right.$ & [22] \\
\hline$Q(H, E)=\frac{a d-b c}{a d+b c}$ & [43] \\
\hline
\end{tabular}

The measures gathered in Table 7, can also be grouped according to their monotonicity with respect to left- and right-hand side probabilities of different perspectives of confirmation. For example, measures $D(H, E)$ and $Z(H, E)$ are monotonic in the Bayesian perspective, measure $M(H, E)$ in the likelihoodist perspective, measure $S(H, E)$ in the strong Bayesian perspective, whereas measures $N(H, E)$ and $F(H, E)$ in the strong likelihoodist perspective.

Let us also observe that the considered measures can be put in some kind of couples according to their definition being inspired by the Bayesian (strong Bayesian) or likelihoodist (strong likelihoodist) perspective. Measure $D(H, E)=\operatorname{Pr}(H \mid E)-\operatorname{Pr}(H)$ can be regarded as a Bayesian counterpart of the likelihoodist measure $M(H, E)=\operatorname{Pr}(E \mid H)-\operatorname{Pr}(E)$. In the same way, we can join measures $S(H, E)$ and $N(H, E)$. Though measure $F(H, E)=[\operatorname{Pr}(E \mid H)-\operatorname{Pr}(E \mid \neg H)] /[\operatorname{Pr}(E \mid H)+\operatorname{Pr}(E \mid \neg H)]$ does not have any popular counterpart, it can paired with newly formulated $F^{\prime}(H, E)=[\operatorname{Pr}(H \mid E)-\operatorname{Pr}(H \mid \neg E)] /[\operatorname{Pr}(H \mid E)+\operatorname{Pr}(H \mid \neg E)]$. Let us also recall that measure $F(H, E)$ is ordinally equivalent to the log likelihood ratio measure $L(H, E)=\log \left[\operatorname{Pr}(E \mid H) /[\operatorname{Pr}(E \mid \neg H)]\right.$, as well as measure $F^{\prime}(H, E)$ is ordinally equivalent to the following Bayesian counterpart of the log likelihood ratio measure $B(H, E)=\log \left[\operatorname{Pr}(H \mid E) /[\operatorname{Pr}(H \mid \neg E)]\right.$. Measures $C(H, E), c_{3}(H, E)$ and $c_{4}(H, E)$ can be regarded as "self adjoint". 
Application of confirmation measures (e.g. all measures from Table 7 except for measure $C(H, E)$ ) for evaluation of rules induced from datasets may lead to obtaining undefined values in particular cases, e.g., for any rule characterized by a contingency table with $a+c=0$ an undefined value of measure $D(H, E)$ is obtained. Such situations are inconvenient and troublesome for data analysts and are generally undesirable. In such situations a measure simply cannot evaluate a rule. In our opinion, the more often undefined values can occur for a measure, the less useful the measure is.

Let us observe, however, that taking advantage of the general definition of the confirmation (2), we can avoid situations when for a particular measure, the answer is undefined. Since the condition $a d-b c=0$ requires that a measure obtains value 0 , we can assume that any measure (despite its actual definition) defaults to 0 whenever $a d-b c=0$. This way, we would not even calculate the value of a measure but basing on the fact that $a d-b c=0$ assign it to 0 . Such an approach would allow us to eliminate some undefined values, e.g., for a contingency table where $a=c=0$, we would obtain an undefined value of measure $D(H, E)$ (the denominator would be equal to 0 ), however using the general definition of confirmation, we see that $a=c=0$ results in the situation of neutrality because $a d-b c=0$, and thus instead of an undefined value we could say that $D(H, E)$ states neutrality.

Let us stress that the above proposition is inspired by practical experiments, in which dealing with undefined values perturbs the rule evaluation procedure. On the other hand, however, valuable procedures of rule induction form rules on the basis of existence of positive examples (and/or counterexamples) in the dataset. In such cases, many of the undefined situations would be avoided as the procedure would not even create rules for which particular measures would be undefined.

\subsection{Properties of selected confirmation measures}

Having established the list of desirable properties of confirmation measures, let us summarize the analyses of measures with respect to them. Table 8 and Table 9 gather the results. Measures $S(H, E), N(H, E), c_{3}(H, E)$ and $c_{4}(H, E)$ are the ones which possess all the properties discussed in this paper which are desirable (i.e. monotonicity $\mathrm{M}$, weak $\mathrm{Ex}_{1}$, weak $\mathrm{L}$ and maximality/minimality, $H S, E S$ and $E H S$ ) and are thus recommended for finding meaningful rules. It is interesting to note that $S(H, E)$ is monotonic in the strong Bayesian perspective of and $N(H, E)$ in the strong likelihoodist perspective, while $c_{3}(H, E)$ and $c_{4}(H, E)$ are obtained combining two confirmation measures, $Z(H, E)$ and $A(H, E)$, monotonic in the Bayesian and in the converse Bayesian perspective. Notice that we considered also confirmation measure $F^{\prime}(H, E)$ that can be interpreted as a strong Bayesian 
counterpart of the strong likelihoodist confirmation measure $F(H, E)$. To the best of our knowledge $F^{\prime}(H, E)$ is a new measure and it has not been considered before. We also included in our considerations Yule's $Q(H, E)$ because it satisfies many interesting properties.

Table 8. Properties of selected measures of confirmation

\begin{tabular}{|c|c|c|c|c|c|c|c|c|c|}
\hline $\begin{array}{l}\text { Confir- } \\
\text { mation } \\
\text { mea- } \\
\text { sure } \\
\end{array}$ & $\begin{array}{l}\text { mono- } \\
\text { toni- } \\
\text { city } \mathrm{M}\end{array}$ & $\mathrm{Ex}_{1}$ & $E x_{1}{ }^{\prime}$ & $\begin{array}{l}\text { weak } \\
\mathrm{Ex}_{1}\end{array}$ & $\mathrm{~L}$ & $\mathrm{~L}^{\prime}$ & $\begin{array}{l}\text { weak } \\
\text { L }\end{array}$ & $\begin{array}{l}\text { maxi- } \\
\text { mality/ } \\
\text { mini- } \\
\text { mality }\end{array}$ & $\begin{array}{l}\text { mono- } \\
\text { tonicity } \\
\text { in the } \\
\text { perspective }\end{array}$ \\
\hline$D(H, E)$ & no & no & no & no & no & no & no & no & Bayesian \\
\hline$M(H, E)$ & no & no & no & no & no & no & no & no & likelihoodist \\
\hline$S(H, E)$ & yes & no & no & yes & no & no & yes & yes & $\begin{array}{l}\text { strong } \\
\text { Bayesian }\end{array}$ \\
\hline$N(H, E)$ & yes & no & no & yes & no & no & yes & yes & $\begin{array}{l}\text { strong } \\
\text { likelihoodist }\end{array}$ \\
\hline$C(H, E)$ & no & no & no & no & no & no & no & no & - \\
\hline$F(H, E)$ & yes & yes & no & yes & yes & no & yes & no & $\begin{array}{l}\text { strong } \\
\text { likelihoodist }\end{array}$ \\
\hline$F^{\prime}(H, E)$ & yes & no & yes & yes & no & yes & yes & no & $\begin{array}{l}\text { strong } \\
\text { Bayesian }\end{array}$ \\
\hline$Z(H, E)$ & yes & yes & no & yes & yes & no & yes & no & Bayesian \\
\hline$A(H, E)$ & yes & no & yes & yes & no & yes & yes & no & $\begin{array}{l}\text { converse } \\
\text { Bayesian }\end{array}$ \\
\hline$c_{1}(H, E)$ & yes & yes & no & yes & no & no & yes & yes & - \\
\hline$c_{2}(H, E)$ & yes & no & yes & yes & no & no & yes & yes & - \\
\hline$c_{3}(H, E)$ & yes & no & no & yes & no & no & yes & yes & - \\
\hline$c_{4}(H, E)$ & yes & no & no & yes & no & no & yes & yes & - \\
\hline$Q(H, E)$ & yes & no & no & yes & yes & yes & yes & no & - \\
\hline
\end{tabular}

Table 9. Symmetry properties of selected measures of confirmation

\begin{tabular}{|c|c|c|c|}
\hline $\begin{array}{l}\text { Confirmation } \\
\text { measure }\end{array}$ & $\begin{array}{l}\text { hypothesis } \\
\text { symmetry } \\
(H S)\end{array}$ & $\begin{array}{l}\text { evidence } \\
\text { symmetry } \\
(E S)\end{array}$ & $\begin{array}{l}\text { evidence- } \\
\text { hypothesis } \\
\text { symmetry }(E H S)\end{array}$ \\
\hline$D(H, E)$ & yes & no & no \\
\hline$M(H, E)$ & no & yes & no \\
\hline
\end{tabular}




\begin{tabular}{|l|l|l|l|}
\hline$S(H, E)$ & yes & yes & yes \\
\hline$N(H, E)$ & yes & yes & yes \\
\hline$C(H, E)$ & yes & yes & yes \\
\hline$F(H, E)$ & yes & no & no \\
\hline$F^{\prime}(H, E)$ & no & yes & no \\
\hline$Z(H, E)$ & yes & no & no \\
\hline$A(H, E)$ & yes & no & no \\
\hline$c_{1}(H, E)$ & yes & no & no \\
\hline$c_{2}(H, E)$ & yes & no & no \\
\hline$c_{3}(H, E)$ & yes & yes & yes \\
\hline$c_{4}(H, E)$ & yes & yes & yes \\
\hline$Q(H, E)$ & yes & yes & yes \\
\hline
\end{tabular}

\section{Conclusions}

Adopting different perspectives for probabilistic relationships between rule's premise and conclusion, one arrives at six formulations of the confirmation concept: four of them (that we call Bayesian confirmation, strong Bayesian confirmation, likelihoodist confirmation and strong likelihoodist confirmation) are well known in the literature, while the other two (that we call converse Bayesian confirmation and converse likelihoodist confirmation) have been introduced in this paper. As observed in [12], [28] with respect to the four classically considered perspectives, and in Section 4.4 of this paper with respect to the two new perspectives, all these perspectives are logically equivalent. Moreover, there exists a general definition of confirmation, expressed in terms of a contingency table rather than in terms of probabilities, that is common for all of them [16]. The general definition has the advantage of being always defined, as opposed to the other considered formulations.

In this paper, we focused our attention on linking qualitative and quantitative aspects of the concept of confirmation. To this end, we introduced an important property of monotonicity of a confirmation measure with respect to left- and right-hand side probabilities of particular perspectives and we checked this property for selected measures. This led us to a conclusion that the six perspectives, although logically equivalent, involve 
different types of monotonicity that differentiate particular confirmation measures. This is due to the observed fact that a measure being strictly monotonic in one perspective is not strictly monotonic in the other perspectives. We have also performed a thorough analysis of several other properties that confirmation measures may possess. They include monotonicity M, $\mathrm{Ex}_{1}, \mathrm{Ex}_{1}$ ', weak $\mathrm{Ex}_{1}, \mathrm{~L}$, L', weak L, maximality/minimality and symmetry properties. Moreover, we discovered and proved what relationships there are between those properties and the monotonicity of a measure in a given perspective. In particular, investigating symmetry properties, we introduced the two new perspectives of converse Bayesian confirmation and converse likelihoodist confirmation showing also that together with the other four previous perspectives they are exhaustive with respect to transformations of confirmation measure related to symmetry properties.

Analysis of chosen confirmation measures with respect to the desirable properties showed that measures $S(H, E), N(H, E), c_{3}(H, E), c_{4}(H, E)$ are particularly valuable.

Further research will focus on experimental exploitation of these conclusions with respect to both association and decision rules.

\section{Acknowledgement}

The first author acknowledges the support from the project PON04a2 $E$ SINERGREEN-RES-NOVAE. The research of the second and third author has been supported by The Polish National Science Centre.

\section{References}

[1] Agrawal, R., Imielinski, T., Swami, A.., 1993. Mining associations between sets of items in massive databases, in: Proc. of the 1993 ACMSIGMOD Int'l Conf. on Management of Data, 207-216.

[2] Bramer, M., 2007. Principles of Data Mining, Springer-Verlag, New York Inc.

[3] Brzezińska, I., Greco, S., Słowiński, R., 2007. Mining Pareto-optimal rules with respect to support and anti-support. Engineering Applications of Artificial Intelligence, vol. 20, no. 5, 587-600.

[4] Carnap, R., 1962. Logical Foundations of Probability, 2nd ed., University of Chicago Press, Chicago. 
[5] Christensen, D., 1999. Measuring confirmation. Journal of Philosophy, vol. $96,437-461$.

[6] Crupi V., Tentori, K., Gonzalez, M., 2007. On Bayesian measures of evidential support: Theoretical and empirical issues. Philosophy of Science, vol. 74, 229-252.

[7] Eells, E., 1982. Rational Decision and Causality. Cambridge University Press, Cambridge.

[8] Eells, E., Fitelson, B., 2002. Symmetries and asymmetries in evidential support. Philosophical Studies, vol. 107, no. 2, 129-142.

[9] Fayyad, U., Piatetsky-Shapiro, G., Smyth, P., 1996. From data mining to knowledge discovery: an overview. In: Fayyad, U., Piatetsky-Shapiro, G., Smyth, P., Uthursamy, R. (eds.) Advances in Knowledge Discovery and Data Mining, AAAI Press, pp. 1-34.

[10] Finch, H.A., 1999. Confirming Power of Observations Metricized for Decisions among Hypotheses. Philosophy of Science, vol. 27, 293-307 and 391-404.

[11] Fitelson, B., 1999. The Plurality of Bayesian Measures of Confirmation and the Problem of Measure Sensitivity. Philosophy of Science, vol. 66, 362-378.

[12] Fitelson, B., 2001. Studies in Bayesian Confirmation Theory. Ph.D. Thesis, University of Wisconsin, Madison.

[13] Fitelson, B., 2006. Logical Foundations of Evidential Support. Philosophy of Science, vol. 73, 500-512.

[14] Fitelson B., 2007. Likelihoodism, Bayesianism, and Relational Confirmation. Synthese, vol. 156, no. 3, 473-489.

[15] Geng, L., Hamilton, H.J., 2006. Interestingness Measures for Data Mining: A Survey. ACM Computing Surveys, vol. 38, no. 3, article 9.

[16] Glass, D.H., 2013. Confirmation measures of association rule interestingness, Knowledge-Based Systems 44, 65-77.

[17] Glass, D.H., 2014. Entailment and symmetry in confirmation measures of interestingness, Information Sciences 279, 552-559.

[18] Greco, S., Pawlak, Z., Słowiński, R., 2004. Can Bayesian confirmation measures be useful for rough set decision rules? Engineering Applications of Artificial Intelligence, vol. 17, no.4, 345-361.

[19] Greco, S., Słowiński, R., Szczęch, I., 2008. Assessing the quality of rules with a new monotonic interestingness measure Z. In: Rutkowski, L., Tadeusiewicz, R., Zadeh, L.A., Zurada, J.M. (eds.), Artificial 
Intelligence and Soft Computing (ICAISC 2008), LNAI, vol. 5097, pp. 556-565. Springer, Heidelberg.

[20] Greco, S., Słowiński, R., Szczęch, I., 2009. Analysis of monotonicity properties of some rule interestingness measures. Control and Cybernetics, vol.38,(1), 9-25.

[21] Greco, S., Słowiński, R., Szczęch, I., 2010. Alternative normalization schemas for Bayesian confirmation measures. In: Hüllermeier, E., Kruse, R., Hoffmann, F. (eds.), Computational Intelligence for Knowledge-Based Systems Design (IPMU 2010), LNAI, vol. 6178, pp. 230-239. Springer-Verlag, Heidelberg.

[22] Greco, S., Słowiński, R., Szczęch, I., 2012. Properties of rule interestingness measures and alternative approaches to normalization of measures. Information Sciences, vol.216, 1-16.

[23] Greco, S., Słowiński, R., Szczęch, I., 2013. Finding Meaningful Bayesian Confirmation Measures. Fundamenta Informaticae, vol.127, 161-176.

[24] Hempel, C.G., 1945. Studies in the logic of confirmation (I). Mind, vol. 54, 1-26.

[25] Kemeny, J., Oppenheim, P., 1952. Degrees of factual support. Philosophy of Science, 19, 307-324.

[26] Kolmogorov, A., 1956. Foundations of Probability (2nd English ed.). Providence, Rhode Island, AMS Chelsea publishing.

[27] Lenca, Ph., Meyer, P., Vaillant, B., Lallich, S., 2008. On selecting interestingness measures for association rules: User oriented description and multiple criteria decision aid. European J. of Operational Research, vol. 184, no. 2, 610-626.

[28] Maher, P., 2005. Confirmation Theory. The Encyclopedia of Philosophy ( $2^{\text {nd }}$ ed.). Macmillan Reference, USA.

[29] McGarry, K., 2005. A survey of interestingness measures for knowledge discovery. The Knowledge Engineering Review, vol. 20, no.1, 39-61.

[30] Michael, E. L., 1920. Marine ecology and the coefficient of association. Journal of Animal Ecology, 8, 54-59.

[31] Mortimer, H., 1988. The Logic of Induction, Paramus, Prentice Hall.

[32] Nicod, J., 1923. Le problème de la logique de l'induction. Alcan, Paris.

[33] Nozick, R., 1981. Philosophical Explanations, Clarendon Press, Oxford (UK). 
[34] Omiecinski, E., 2003. Alternative interest measures for mining associations in databases. IEEE Transactions on Knowledge and Data Engineering, vol. 15, no. 1, 57-69.

[35] Pałkowski, Ł., Błaszczyński, J., Skrzypczak, A., Błaszczak, J., Kozakowska, K., Wróblewska, J., Kożuszko, S., Gospodarek, E., Krysiński, J., Słowiński, R., 2014. Antimicrobial Activity and SAR Study of New Gemini Imidazolium-based Chlorides. Chemical Biology \& Drug Design, 83 no.3, 278-288.

[36] Pearson, K., 1900. Mathematical contributions to the theory of evolution. VII On the correlation of characters not quantitatively measurable. Philos. Trans. R. Soc. Lond.Ser. A Math. Phys. Eng. Sci., 195, 1-47.

[37] Piatetsky-Shapiro, G., 1991. Discovery, analysis, and presentation of strong rules. Chapter 12, in: Knowledge Discovery in Databases, AAAI/MIT Press.

[38] Rips, L.J., 2001. Two Kinds of Reasoning. Psychological Science, vol. $12,129-134$.

[39] Silberschatz, A., Tuzhilin, A., 1996. What Makes Patterns Interesting in Knowledge Discovery Systems. IEEE Transactions on Knowledge and Data Engineering, vol. 8, no. 6, 970-974.

[40] Szczęch I., 2009. Multicriteria Attractiveness Evaluation of Decision and Association Rules. Transactions on Rough Sets X, LNCS series, vol. 5656, Springer, Berlin, pp.197-274.

[41] Tan, P.-N., Kumar, V., Srivastava, J., 2002. Selecting the right interestingness measure for association patterns. In: Proc. of the $8^{\text {th }}$ Int. Conf. on Knowledge Discovery and Data Mining (KDD 2002). Edmonton, Canada, pp.32-41.

[42] Tan, P.-N., Kumar, V., Srivastava, J., 2004. Selecting the right objective measure for association analysis. Information Systems, vol. 29, no. 4, 293-313.

[43] Yule, G. U., 1900. On the association of attributes in statistics. Philosophical Transactions of the Royal Society, A, 75, 257-319.

[44] Yule, G. U., 1912. On the methods of measuring the association between two attributes. J. Roy. Statist. Soc., 75, 579-652. 